ARTICLE

\title{
Temperature sensitivity of Notch signaling underlies species-specific developmental plasticity and robustness in amniote brains
}

\author{
Tadashi Nomura (10 ${ }^{1 凶}$, Kohjiro Nagao ${ }^{2,3}$, Ryo Shirai ${ }^{4,5}$, Hitoshi Gotoh ${ }^{1}$, Masato Umeda (iD ${ }^{2,6}$ \& Katsuhiko Ono
}

\begin{abstract}
Ambient temperature significantly affects developmental timing in animals. The temperature sensitivity of embryogenesis is generally believed to be a consequence of the thermal dependency of cellular metabolism. However, the adaptive molecular mechanisms that respond to variations in temperature remain unclear. Here, we report species-specific thermal sensitivity of Notch signaling in the developing amniote brain. Transient hypothermic conditions increase canonical Notch activity and reduce neurogenesis in chick neural progenitors. Increased biosynthesis of phosphatidylethanolamine, a major glycerophospholipid components of the plasma membrane, mediates hypothermia-induced Notch activation. Furthermore, the species-specific thermal dependency of Notch signaling is associated with developmental robustness to altered Notch signaling. Our results reveal unique regulatory mechanisms for temperature-dependent neurogenic potentials that underlie developmental and evolutionary adaptations to a range of ambient temperatures in amniotes.
\end{abstract}

\footnotetext{
${ }^{1}$ Developmental Neurobiology, Kyoto Prefectural University of Medicine, INAMORI Memorial Building, 1-5 Shimogamo-Hangi cho, Sakyo-ku, Kyoto 6060823, Japan. ${ }^{2}$ Department of Synthetic Chemistry and Biological Chemistry, Graduate School of Engineering, Kyoto University, Katsura, Nishikyo-ku, Kyoto 615-8510, Japan. ${ }^{3}$ Department of Biophysical Chemistry, Kyoto Pharmaceutical University, 5 Misasaginakauchi-cho, Yamashina-ku, Kyoto 607-8414, Japan. ${ }^{4}$ School of Medicine, Niigata University, 757 Ichibancho, Asahimachi-dori, Chuo Ward, Niigata City 951-8510, Japan. ${ }^{5}$ Japanese Red Cross Society Kyoto Daini Hospital, 355-5 Haru-obi cho, Marutamachi- noboru, Kamaza-dori, Kamigyo-ku, Kyoto 602-8026, Japan. ${ }^{6}$ HOLO BIO Co., Ltd. 1-36 Goryo Ohara,

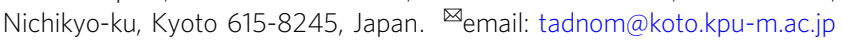


T he complex interplay of genes and the environment is responsible for the emergence of constant and variable traits in organisms. Canalization and phenotypic plasticity, which represent developmental robustness and flexibility in response to genetic and environmental perturbations, are thought to play critical roles in the control of phenotypic stability and divergence $^{1-4}$. Understanding the regulatory mechanisms underlying these variable phenotypic outcomes is necessary to clarify the intrinsic forces that spur adaptive evolution in a wide range of environmental conditions.

Ambient temperature significantly influences the developmental programs of both ectothermic and endothermic animals ${ }^{5-7}$. In general, lower temperatures decelerate developmental timing in egg-laying (oviparous) species, which has been assumed to result from the thermal dependency of cellular metabolism ${ }^{8,9}$; for example, departures from the optimum temperatures of biochemical reactions prolong cell divisions and differentiation. However, several species exhibit remarkable plasticity to temperature variations ${ }^{5}$. With only a few exceptions $s^{10-12}$, our knowledge of the adaptive molecular mechanisms that underlie the thermal sensitivity and robustness of developmental signaling is still limited.

Notch signaling has been well documented as an important regulator of cell fate decisions during embryogenesis ${ }^{13}$. Canonical Notch signaling is activated by the binding of membrane-bound ligands (Delta, Serrate, and Lag2) to the Notch receptor, which triggers receptor cleavage and the translocation of the Notch intracellular domain (NICD) into the nucleus. Within the nucleus, the NICD forms a transcriptional regulatory complex with other coactivators that controls downstream gene expression. In the developing central nervous system, Notch signaling plays a crucial role in neurogenesis in a variety of species ${ }^{13,14}$ : the loss of Notch signaling accelerates neuronal differentiation, while increased Notch signaling preserves neural progenitor pools and suppresses neurogenesis ${ }^{15,16}$. In contrast to the severe phenotypes produced by experimental manipulations, physiological levels of Notch signaling are highly stable to variable temperatures ${ }^{17}$. In Drosophila, the net level of Notch signaling is regulated by temperature-dependent compensatory changes in the flux of endocytic trafficking networks ${ }^{18}$. However, the evolutionary conservation and diversification of the thermal sensitivity of Notch signaling and the associated phenotypic influences remain to be elucidated.

Here, we report species-specific thermal dependency of Notch signaling in the neural progenitors of developing amniote brains. Transient hypothermic incubation significantly increases canonical Notch activity in chick neural progenitors, which is mediated by ligand ubiquitylation and internalization. We found that temperature-dependent changes in the biosynthesis and cell surface exposure of phosphatidylethanolamine (PE), a major membrane glycerophospholipid, contribute to the thermal responsiveness of Notch signaling in chick neural progenitors. In contrast, Notch signaling levels are highly stable to temperature variations in developing mouse neocortical progenitors, which provides a safeguard of Notch-dependent neuronal migration during mammalian-specific neocortical development. Our results revealed that the temperature-dependent regulation of Notch signaling is tightly coupled with species-specific neurogenic programs, which underlies multiple levels of developmental canalization in response to environmental changes during amniote evolution.

\section{Results}

Inverse temperature dependency of Notch signal in nonmammals. To investigate the temperature dependency of Notch activities in developing reptilian and avian brains, we isolated neural progenitors from the pallium of turtle and chick embryos and incubated them for $24 \mathrm{~h}$ at $30^{\circ} \mathrm{C}$ (an optimal temperature for reptilian embryogenesis, but hypothermic for Aves) or $37^{\circ} \mathrm{C}$ (normothermic for avian embryogenesis, but hyperthermic for reptiles). To monitor Notch activity under different temperature conditions, a Notch reporter vector (4xCSL-luc) was introduced into isolated neural progenitors prior to culture (Fig. 1a, b). We found inverse temperature dependence of Notch activity in the two species: culture at $30^{\circ} \mathrm{C}$ significantly increased Notch reporter activities in turtle and chick neural progenitors relative to the culture at $37^{\circ} \mathrm{C}$ (Fig. 1c, d). The $\gamma$-secretase inhibitor DAPT completely blocked Notch activity in chick neural progenitors at $30^{\circ} \mathrm{C}$, suggesting that hypothermia-induced Notch activity is mediated by Notch receptor cleavage (Fig. 1e). Accordingly, the low temperature increased the abundance of cleaved forms of the Notch1 receptor (Notch intracellular domain: NICD) in cultured chick neural progenitors (Fig. 1f). In contrast, Notch activity was lower in chicken liver primary cells, heart muscle cells, and DF-1 fibroblast cells at $30^{\circ} \mathrm{C}$ than at $37^{\circ} \mathrm{C}$ $($ Fig. S1a-c). These data suggest that increased Notch activity at lower temperatures is unique to the neural progenitors of oviparous amniotes.

Next, we examined the impact of temperature shifts on pallial neurogenesis in developing chick embryos. Fertilized chicken eggs incubated at $37^{\circ} \mathrm{C}$ until E5 (Hamburger and Hamilton stages 24/ 25) were shifted to $30^{\circ} \mathrm{C}$ (hypothermic for chick embryogenesis) for $24 \mathrm{~h}$, when massive neurogenesis occurs in the developing pallium. Neural progenitors residing in the ventricular zone was labeled by administration of a small amount of 5-ethynyl-2'deoxyuridine (EdU) or the electroporation of GFP expression vector prior to the temperature shift (Figs. $1 \mathrm{~g}$ and S1i). To examine S-phase re-entry of neural progenitors under different temperatures, ventricular cells were sequentially labeled with GFP expression vector and EdU at different timing (Fig. S1i). Hypothermia significantly delayed the progression of developmental stages of chicken embryos (Fig. Sid-f). Although the number of phosphorylated histone H3-positive mitotic cells in the pallium was comparable (Fig. S1g,h), the proportion of S-phase re-entry among ventricular progenitors was reduced under hypothermic conditions (Fig. S1j, k, m). Furthermore, the number of Sox2-negative cells among the EdU-labeled cells in the pallial neuronal zone was significantly reduced at $30^{\circ} \mathrm{C}$ relative to that at $37^{\circ} \mathrm{C}$ (Fig. 1h-l). These data suggested that the lower temperature specifically suppressed cell cycle progression and neuronal differentiation in the developing chick pallium.

To determine whether the reduced neuronal differentiation observed in chick embryos under hypothermic conditions was due to the augmented activity of Notch signaling, we tried to block Notch signaling by using a dominant-negative form of Mastermind like transcriptional coactivator 1 (DN-MAML1) that interferes with Notch-dependent transcriptional activity ${ }^{19}$. We electroporated a GFP expression vector either alone or together with a vector expressing DN-MAML1 into the developing chick pallium and incubated the embryos for $24 \mathrm{~h}$ under normothermic and hypothermic conditions (Fig. $1 \mathrm{~m}$ ). Consistent with previous results, low temperature significantly reduced the number of GFP-labeled neurons and maintained neuronal progenitors in the ventricular zone, while the hypothermia-dependent reduction in neuronal differentiation was completely restored by the overexpression of DN-MAML1 (Fig. 1n,o). Conversely, the overexpression of NICD at $37^{\circ} \mathrm{C}$ significantly reduced neuronal differentiation and cell cycle re-entry in the developing chick pallium (Figs. 1n, o and S1l, m). These data indicate that the thermal sensitivity of Notch signaling underlies the temperature dependency of neurogenesis in the developing chick pallium. 


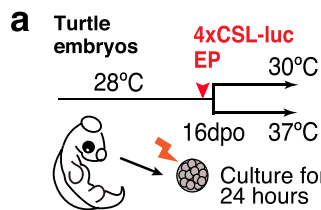

C

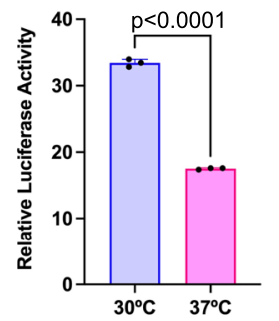

Turtle neural progenitors
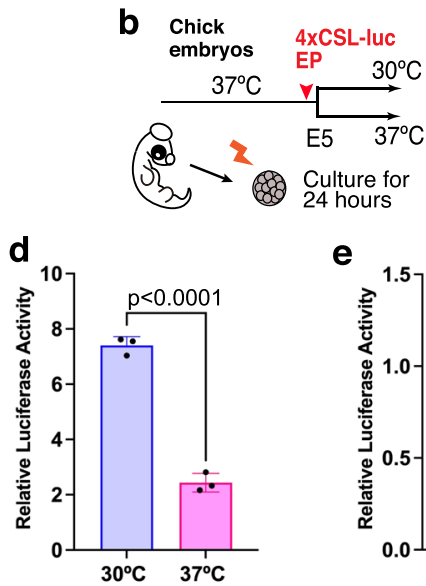

Chick neural progenitors
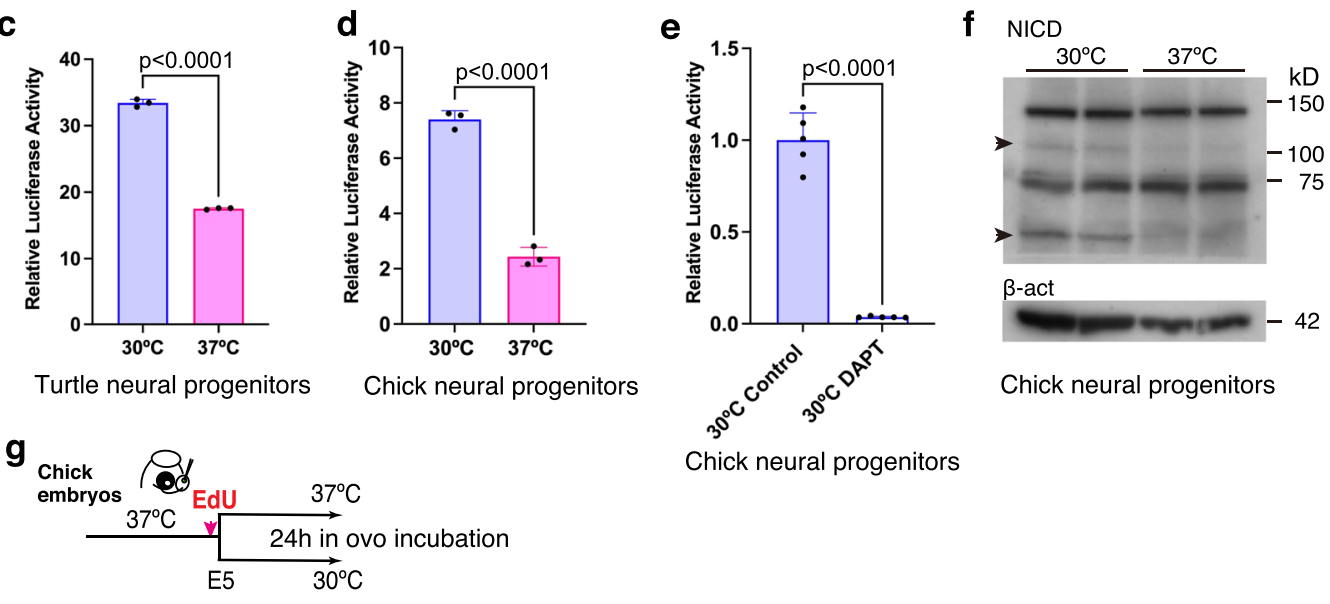

Chick neural progenitors

Chick neural progenitors
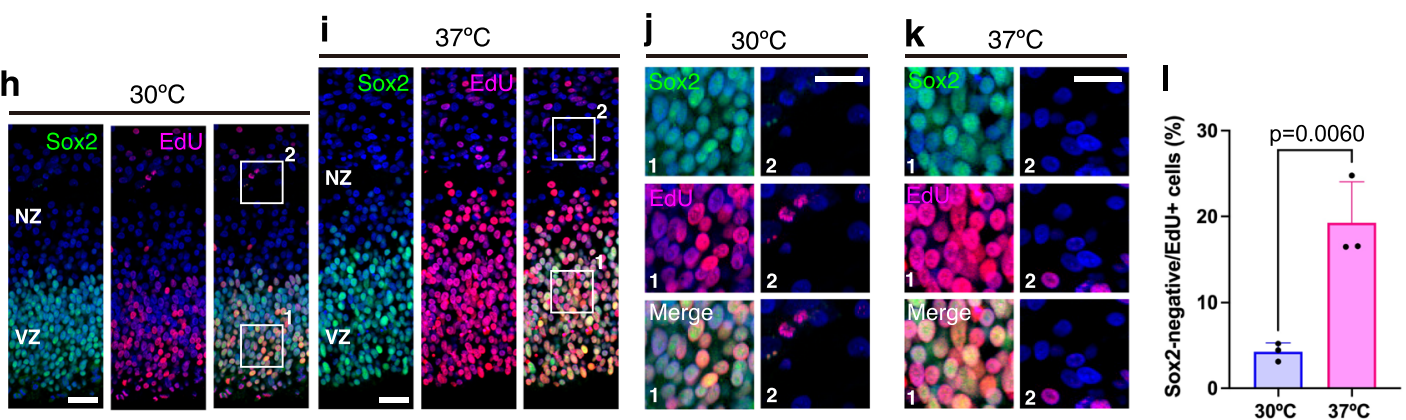

m

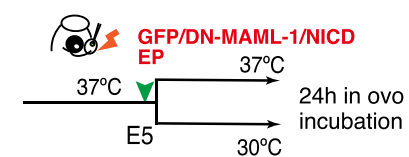

n
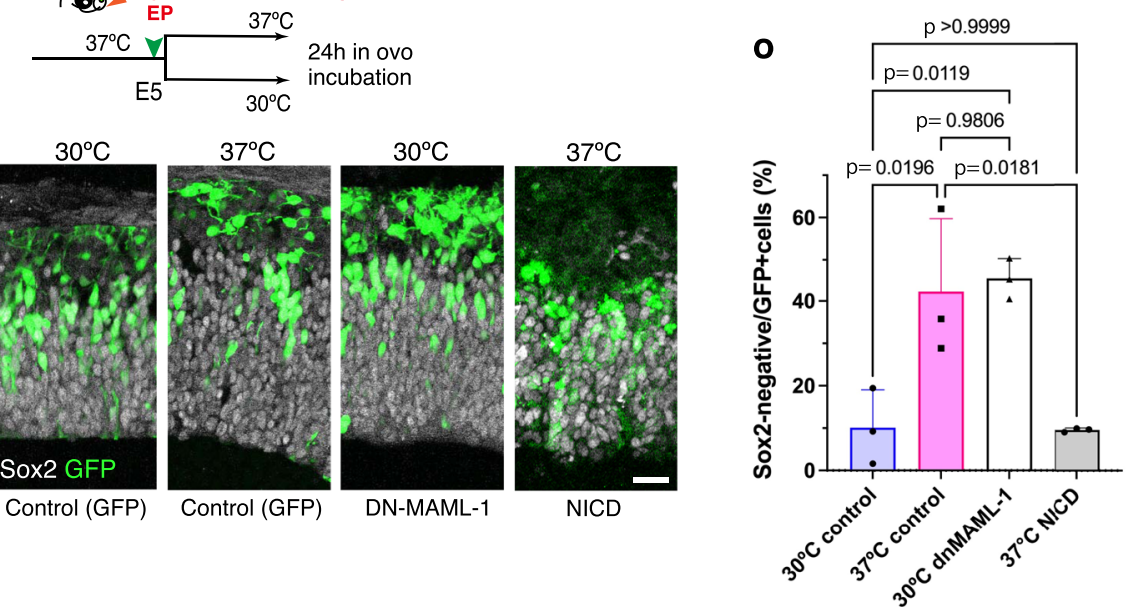

Endocytosis mediates hypothermia-induced Notch activation. We explored the potential mechanisms underlying the inverse temperature dependency of Notch activation in developing chick neural progenitors. Notch activity induced by the overexpression of NICD did not differ between normothermic and hypothermic conditions (Fig. 2a), indicating that NICD-dependent transcriptional regulation is not temperature-sensitive. We also examined the expression levels of genes encoding secretase components that are responsible for Notch receptor cleavage. RNA-seq analysis indicated that the expression of ADAM metallopeptidase domain 10 (ADAM10) and Presenilin1 (PSEN1), major components of $\alpha$ - and $\gamma$-secretases, respectively, were considerably lower in neural progenitors cultured at $30^{\circ} \mathrm{C}$ than in those cultured at $37^{\circ} \mathrm{C}$ (Fig. S2a), suggesting that hypothermia-induced Notch activation is not due to increased enzymatic activities related to receptor processing. Next, we examined the expression profiles of mRNAs encoding the Notch signaling components in cultured neural progenitors. Hypothermia significantly increased the expression levels of genes encoding Notch receptor (Notch1) and ligands, such as Delta like 4 (Dll4), Jagged 1(Jag1), and Jagged 4 (Jag4), as well as the downstream target gene Hes1 (Fig. 2b). To determine whether hypothermia-induced Notch activation is due to 
Fig. 1 Inverse temperature dependency of Notch activity in neural progenitors of oviparous animals. a, b Monitoring Notch activities in turtle (a) and chick (b) neural progenitors cultured at $30^{\circ} \mathrm{C}$ or $37^{\circ} \mathrm{C}$ for $24 \mathrm{~h}$. A Notch reporter vector (4xCSL-luc) was introduced to isolated neural progenitors prior to culture. c, d Notch reporter activities in turtle (c) and chick (d) neural progenitors cultured at $30^{\circ} \mathrm{C}$ or $37^{\circ} \mathrm{C}$ (mean $+\mathrm{SD}, n=3$ biologically independent samples in each group). e DAPT treatment suppressed Notch activity in chicken neural progenitors cultured at $30^{\circ} \mathrm{C}$ (means $+\mathrm{SD}, n=5$ biologically independent samples in each group). $\mathbf{f}$ NICD contents of chick neural progenitors cultured at different temperatures. $\mathbf{g}$ Schematic illustration of the temperature shift experiment in developing chick embryos. h-k Distribution of Sox2 and/or EdU-positive cells in the developing chick pallium incubated at $30^{\circ} \mathrm{C}(\mathbf{h}, \mathbf{j})$ or $37^{\circ} \mathrm{C}(\mathbf{i}, \mathbf{k})$. Insets represent Sox2-positive or negative EdU-labeled cells. I The proportion of Sox2-negative and EdU-positive cells in the chick pallium incubated at different temperatures (means $+\mathrm{SD}, n=3$ biologically independent samples in each group). $\mathbf{m}$ Schematic illustration of DNMAML1 or NICD overexpression in the developing chick pallium. $\mathbf{n}$ Distribution of GFP-positive cells in GFP control, DN-MAML1- or NICD-overexpressing chick pallium incubated at $30^{\circ} \mathrm{C}$ or $37^{\circ} \mathrm{C}$. o The proportion of Sox2-negative and GFP-positive cells in controls $\left(30^{\circ} \mathrm{C}\right.$ or $37^{\circ} \mathrm{C}$ ), and DN-MAML1- or NICD-overexpressing chick pallium at $30^{\circ} \mathrm{C}$ or $37^{\circ} \mathrm{C}$ (means $+\mathrm{SD}, n=3$ biologically independent samples in each group). Two-sided, unpaired $t$-test for $\mathbf{c}, \mathbf{d}, \mathbf{e}$, and $\mathbf{I}$; ordinary one-way ANOVA for $\mathbf{o}$ ( $p$-values were adjusted by Tukey test for multiple comparisons). P-values are indicated in each graph. Scale bars: $25 \mu \mathrm{m}$.
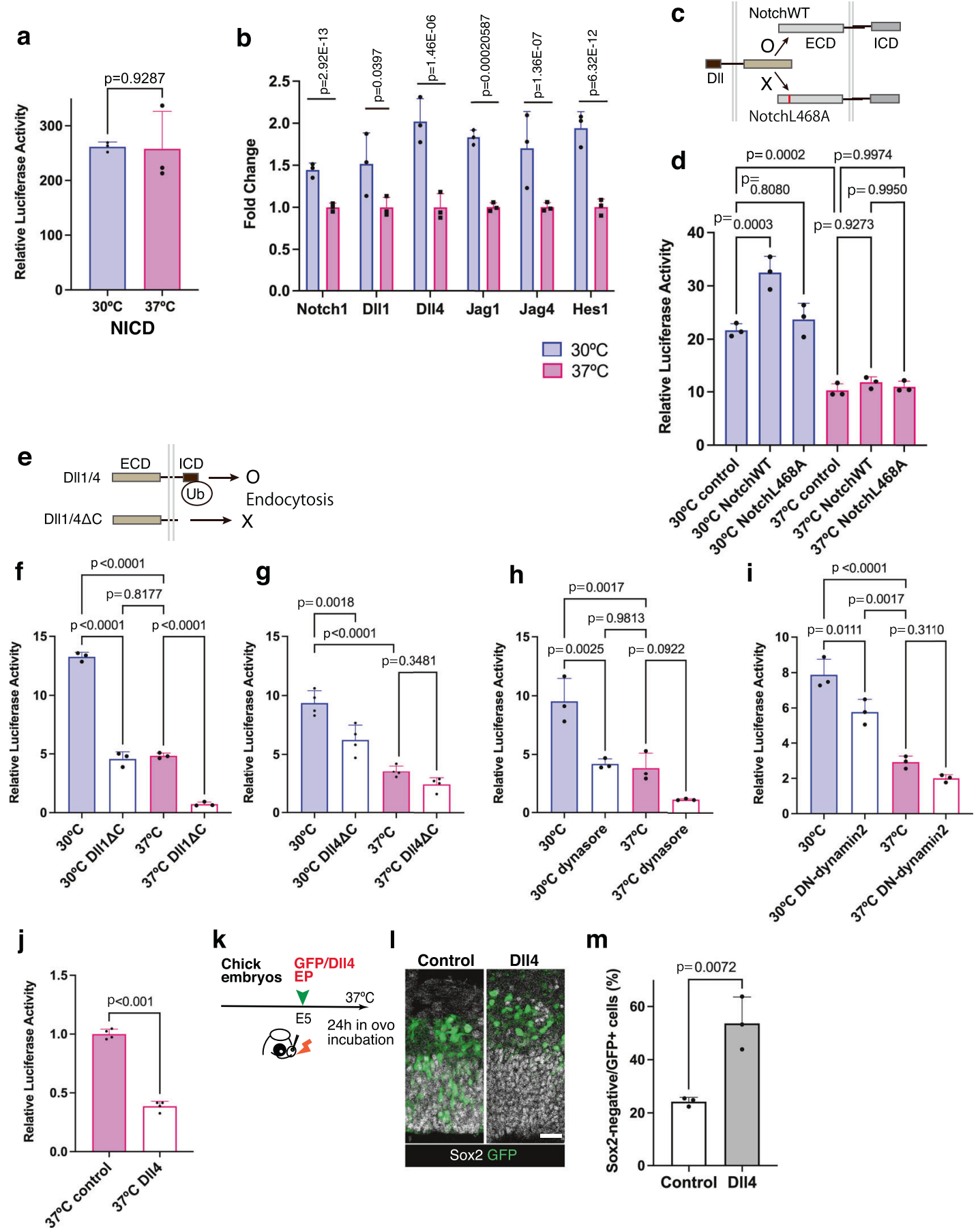

m

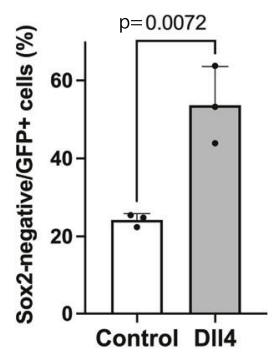


Fig. 2 Ligand endocytosis mediates temperature-dependent Notch activity. a NICD-dependent Notch reporter activity in chick neural progenitors cultured at $30^{\circ} \mathrm{C}$ or $37^{\circ} \mathrm{C}$ (mean $+\mathrm{SD}, n=3$ biologically independent samples in each group). b Relative expression levels of genes encoding Notch signaling components in chick neural progenitors cultured at $30^{\circ} \mathrm{C}$ or $37^{\circ} \mathrm{C}$ (mean $+\mathrm{SD}, n=3$ biologically independent samples in each group).

c Overexpression of Notch1 (NotchWT) or mutant Notch (NotchL468A) receptors in developing chick neural progenitors. $\mathbf{d}$ Notch reporter activities in control, NotchWT, and NotchL468A overexpressing samples cultured at $30^{\circ} \mathrm{C}$ or $37^{\circ} \mathrm{C}$ (mean $+\mathrm{SD}, n=3$ biologically independent samples in each group). e Schematic drawing of DII1/4 and DII1/4 C ; the latter lacks the intracellular domain (ICD) required for ubiquitylation and endocytosis. $\mathbf{f}$, $\mathbf{g}$ Notch reporter activity in controls and D\|l1 $1 \mathrm{C}$ - (f) or D\| $4 \Delta \mathrm{C}(\mathbf{g})$-overexpressing neural progenitors cultured at $30^{\circ} \mathrm{C}$ or $37^{\circ} \mathrm{C}[\mathrm{mean}+\mathrm{SD}, n=3(\mathbf{f})$ and 4 (g) biologically independent samples in each group]. $\mathbf{h}$ Notch reporter activity in control and dynasore-treated neural progenitors cultured at $30^{\circ} \mathrm{C}$ or $37^{\circ} \mathrm{C}$ (mean $+\mathrm{SD}, n=3$ biologically independent samples in each group). i Notch reporter activity in control and dominant-negative dynamin 2 (DNdynamin 2) overexpressing neural progenitors cultured at $30^{\circ} \mathrm{C}$ or $37^{\circ} \mathrm{C}$ (mean $+\mathrm{SD}, n=3$ biologically independent samples in each group). $\mathbf{j}$ Notch reporter activity in control and DII4-overexpressing neural progenitors cultured at $37^{\circ} \mathrm{C}$ (mean $+\mathrm{SD}, n=4$ biologically independent samples in each group). $\mathbf{k}$ Schematic illustration of GFP or Dll4 overexpression in the developing chick pallium. I Distribution of Sox2 and/or GFP-positive cells in the developing chick pallium in control and DII4 electroporated samples. $\mathbf{m}$ The proportion of Sox2-negative and GFP-positive cells in controls and DII4overexpressing chick pallium at $37^{\circ} \mathrm{C}$ (means $+\mathrm{SD}, n=3$ biologically independent samples in each group). Two-sided, unpaired $t$-test for $\mathbf{a}, \mathbf{j}$, and $\mathbf{m}$ ( $p$-values are indicated in each graph), two-sided, negative bimodal distribution for $\mathbf{b}$ ( $p$-values were adjusted for multiple comparisons by using Benjamin-Hochberg method, FDR of DII1 is not true), ordinary one-way ANOVA for $\mathbf{d}$ and $\mathbf{f}-\mathbf{i}$ ( $p$-values were adjusted by Tukey test for multiple comparisons). Scale bar: $25 \mu \mathrm{m}$.

increased receptor expression, we overexpressed the Notch1 receptor in chick neural progenitors. The introduction of the Notch1 receptor did not increase Notch activity under normothermic conditions, while it further increased hypothermiadependent Notch activity under hypothermic conditions. In contrast, a mutant Notch1 receptor (NotchL468A) that was unable to bind ligands ${ }^{20}$ did not facilitate Notch activity under either normothermic or hypothermic conditions (Fig. 2c,d). Thus, although the increase in Notch receptor expression was not sufficient to reproduce hypothermia-induced Notch activation, ligand-dependent Notch receptor activity was facilitated by a lower temperature in developing chicken neural progenitors. To further confirm the requirement of endogenous ligands for temperature-dependent Notch activity, we introduced a synthetic Notch receptor (SynNotch) that was designed to reproduce Notch-dependent cellular reactions in response to artificial ligands ${ }^{21}$. Although the ligand-dependent activation of SynNotch signaling was recapitulated in cultured chicken neural progenitors, we could not detect a hypothermia-dependent increase in reporter activity with an artificial ligand (Fig. S2b), suggesting that the temperature sensitivity of Notch signaling depends on the properties of the endogenous ligands in developing chicken neural progenitors.

To investigate the distribution of endogenous ligands in chicken neural progenitors under different temperature conditions, we determined the cellular localization of the Dll ligand by a cell surface biotinylation assay. Under hypothermic conditions, the relative amount of the Dll ligand on cell surface was decreased, while it was enriched in the intracellular fractions, suggesting that lower temperature increased ligand internalization, rather than cell surface representation (Fig. S2c,d). Accordingly, hypothermia facilitated the ubiquitylation of the Dll ligand (Fig. S2e), which is essential for ligand internalization. Furthermore, the overexpression of Dll1 or Dll4 lacking the intracellular domain responsible for ubiquitylation (Dll1 $\Delta \mathrm{C}$ or Dll4 $\Delta \mathrm{C}$ ) suppressed hypothermia-induced Notch activity in cultured chick neural progenitors (Fig. $2 \mathrm{f}, \mathrm{g}$ ), corroborating the requirement of ligand ubiquitylation and internalization for hypothermiadependent Notch activation.

Several studies have shown that the endocytosis of Notch ligands is a prerequisite for Notch signaling activation ${ }^{22}$. We confirmed that the pharmacological blockade of dynamindependent endocytosis significantly suppressed temperaturedependent Notch activity in chicken neural progenitors (Fig. 2h). Furthermore, hypothermia-induced Notch activity was reduced by dominant-negative dynamin 2 (Fig. 2i), but not by dominantnegative dynamin 1 (Fig. S2f), suggesting that dynamin 2-dependent ligand endocytosis ${ }^{23}$ is critical for the enhancement of Notch activity in developing chicken neural progenitors under hypothermic conditions.

To examine whether the increased expression of Notch ligands is sufficient to activate Notch signaling, we overexpressed Dll4 in chick neural progenitors. However, relative to the controls, the introduction of Dll4 decreased Notch activity at $37^{\circ} \mathrm{C}$ (Fig. 2j). We also confirmed that the electroporation of Dll4 increased the proportion of Sox2-negative cells in the developing chick pallium (Fig. $2 \mathrm{k}-\mathrm{m}$ ), in accord with the previous finding that Dll ligands reduce Notch activity and facilitate neuronal differentiation ${ }^{24}$. These data suggest that the increased expression of Notch ligands is not sufficient to increase Notch activity, and additional mechanisms might underlie the temperature sensitivity of Notch signaling in developing chick neural progenitors.

Hypothermia increases specific phospholipids in chick. To reveal the potential mechanisms underlying the inverse temperature dependency of Notch activity in chick neural progenitors, we sought to determine the contribution of other biological pathways under hypothermic conditions. Hypothermia-induced global changes in transcriptome components involved in multiple metabolic pathways in cultured chick neural progenitors (Fig. S3a, b). We focused on the enrichment of cellular components associated with the membrane-enclosed lumen in response to the temperature change (Fig. S3a). Several studies have shown that temperature-dependent changes in membrane lipid compositions confer thermal adaptability by modulating the physical properties of plasma membranes and membrane-associated cellular events ${ }^{25-28}$. Accordingly, we performed mass spectrometry to compare the major phospholipid components of chicken neural progenitors under normothermic and hypothermic culture conditions. Notably, hypothermic culture increased specific molecular species of phosphatidylethanolamine (PE) containing shorter acyl chains and fewer double bonds (Figs. 3a and S4a). Product ion scan analysis by LC-MS/MS showed that most of the PE species affected by hypothermia consisted of C16-18 saturated or unsaturated fatty acids with one double bond (Fig. 3c-e). Conversely, the abundance of specific species of phosphatidylcholine with similar fatty acid characteristics to the increased PE species was reduced by hypothermic culture conditions (Figs. 3b, f-h and S4b), suggesting that hypothermia induced the de novo synthesis of PE at the expense of PC production.

There are two major metabolic pathways involved in $\mathrm{PE}$ biosynthesis: the CDP-ethanolamine pathway and the phosphatidylserine decarboxylase (Psd) pathway ${ }^{29}$. The RNA-seq analysis of chicken neural progenitors identified hypothermia-dependent 

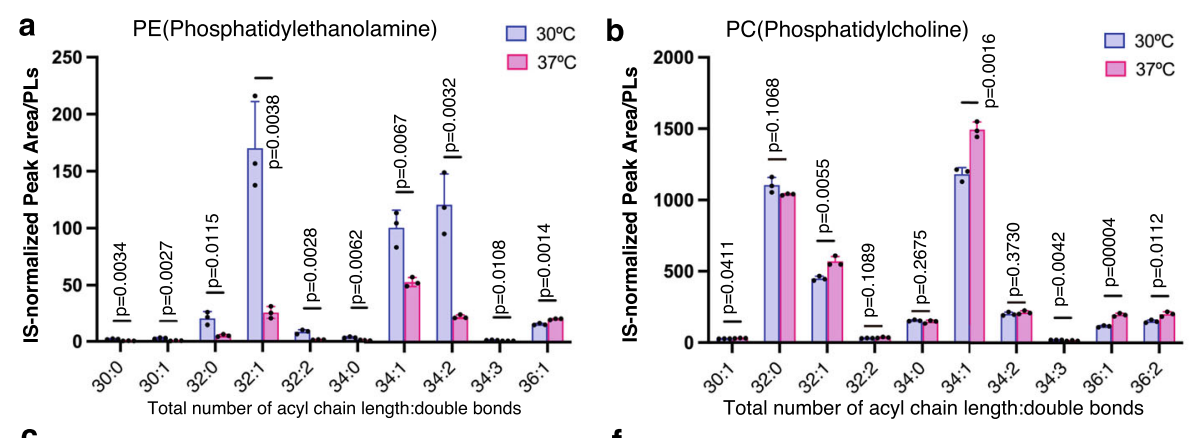

C

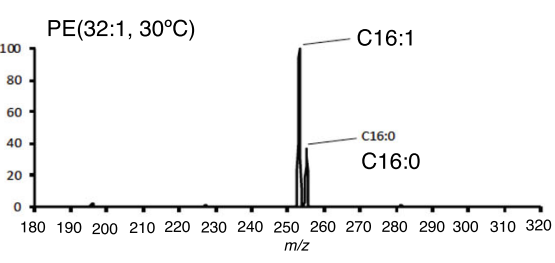

f

d
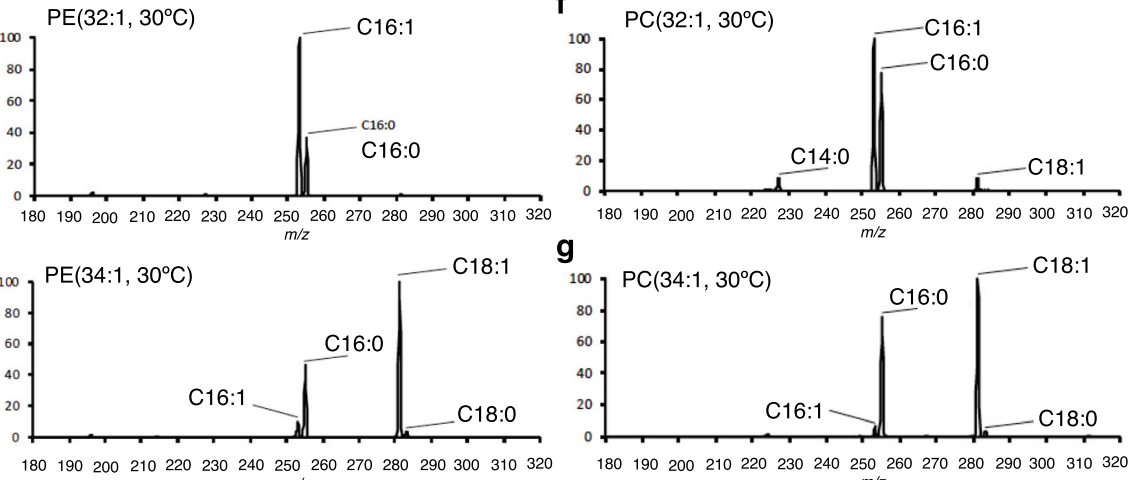

9

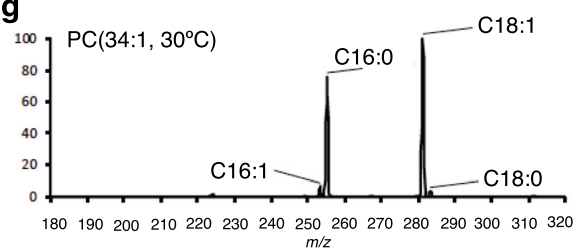

e
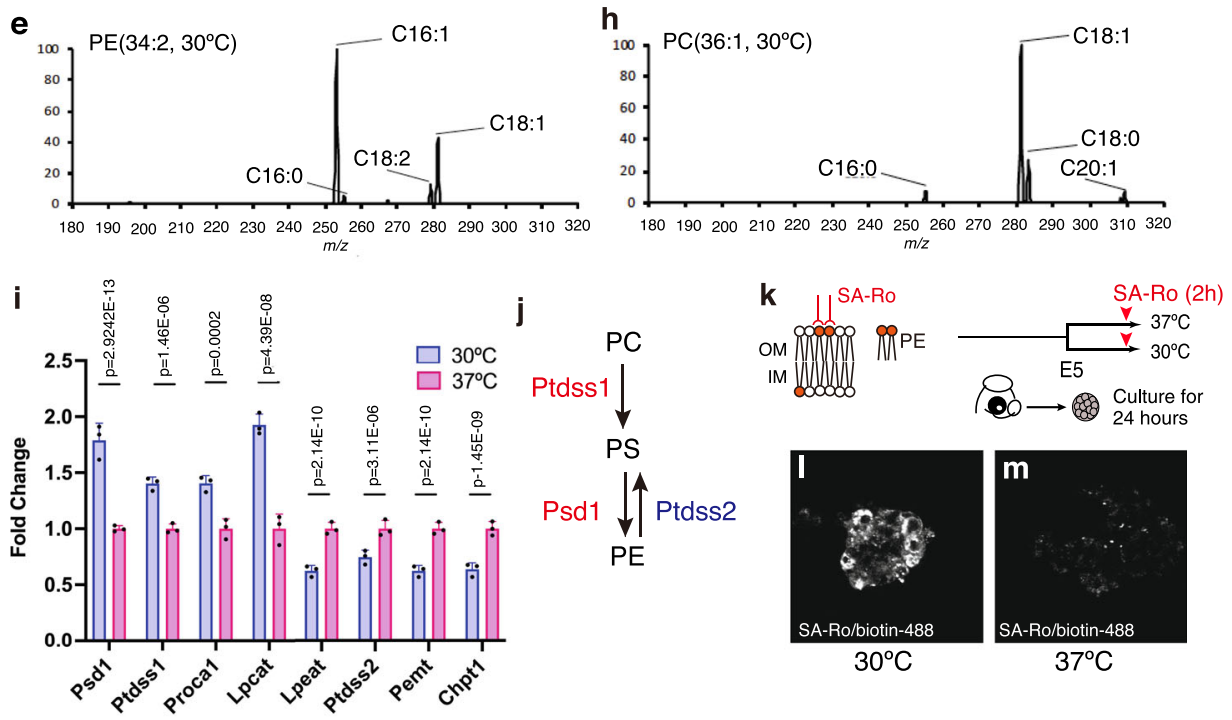

Fig. 3 Enrichment of cell surface PE mediates Notch signaling. a, b PE (a) and PC (b) molecule contents of chick neural progenitors cultured at $30{ }^{\circ} \mathrm{C}$ or $37^{\circ} \mathrm{C}$ (mean + SD, $n=3$ biologically independent samples in each group). c-h Fatty acid composition of PE (32:1, c; 34:1, d; 34:2, e) or PC (32:1, f; 34:1, g; $36: 1, \mathbf{h}$ ) in chick neural progenitors cultured at $30^{\circ} \mathrm{C}$. i Relative expression levels of enzymes involved in PE synthesis in chick neural progenitors cultured at $30^{\circ} \mathrm{C}$ or $37^{\circ} \mathrm{C}$ (mean $+\mathrm{SD}, n=3$ biologically independent samples in each group). $\mathbf{j}$ Biosynthesis of PE through the Psd pathway. $\mathbf{k}$ Labeling of cell surface PE by SA-Ro treatment in nonpermeable condition. I, $\mathbf{m ~ S A - R o ~ f l u o r e s c e n t ~ l a b e l i n g ~ o f ~ c h i c k ~ n e u r a l ~ p r o g e n i t o r s ~ c u l t u r e d ~ a t ~} 30^{\circ} \mathrm{C}(\mathbf{I})$ or $37^{\circ} \mathrm{C}$ ( $\mathbf{m}$ ). Two-sided, unpaired $t$-test for $\mathbf{a}, \mathbf{b}$, two-sided, negative bimodal distribution for $\mathbf{i}$ ( $p$-values were adjusted for multiple comparisons by using Benjamin-Hochberg method). P-values are indicated in each graph.

increases in phosphatidylserine decarboxylase 1 (Psd1) and phosphatidylserine synthase 1 (Ptdss1) but a decrease in Ptdss2, suggesting that hypothermia facilitated the directional biosynthesis of PE through the Psd pathway (Fig. 3i, j). In contrast, we could not detect altered expression of genes associated with the CDP-ethanolamine pathway, such as ethanolaminphosphotransferase 1 (EPT1L; deposited to Mendeley Data: DOI: 10.17632/ 9zxt47grjf.2).

To examine the cellular distribution of $\mathrm{PE}$ in developing chicken neural progenitors, we utilized a streptavidin-conjugated peptide probe (SA-Ro) that specifically binds to $\mathrm{PE}^{30}$. $\mathrm{PE}$ is usually enriched on the inner leaflet of plasma membranes, while it is exposed to cell surfaces during specific cellular events ${ }^{30-33}$ which can be detected by using a peptide probe under nonpermeabilized conditions (Fig. 3k). We found that hypothermia dramatically increased the SA-Ro-mediated fluorescent labeling of chicken neural progenitors (Fig. 3l, $\mathrm{m}$ ), suggesting that an increased amount of PE was exposed on the cell surface under hypothermic conditions. Temperature-dependent changes in PE and PC contents were also evident in cultured turtle neural progenitors, although the impact of temperature variations was much weaker than that in chick neural progenitors (Fig. S4c, d). Thus, the thermal sensitivity of phospholipid synthesis might be shared among oviparous amniotes. 
a

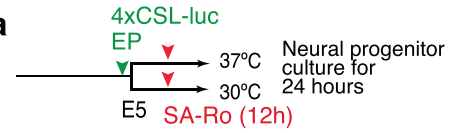

b

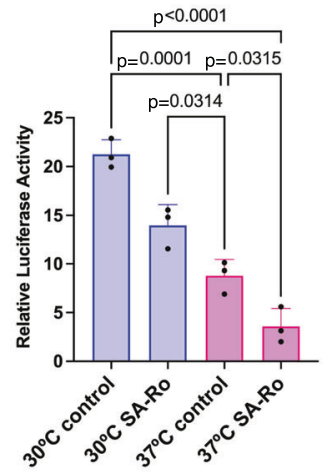

C
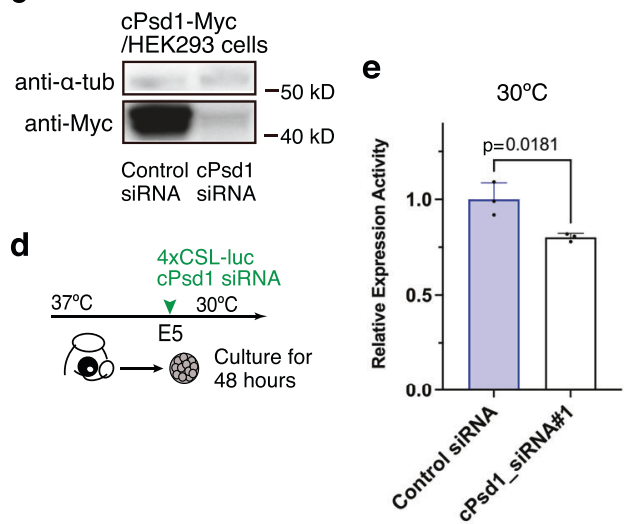

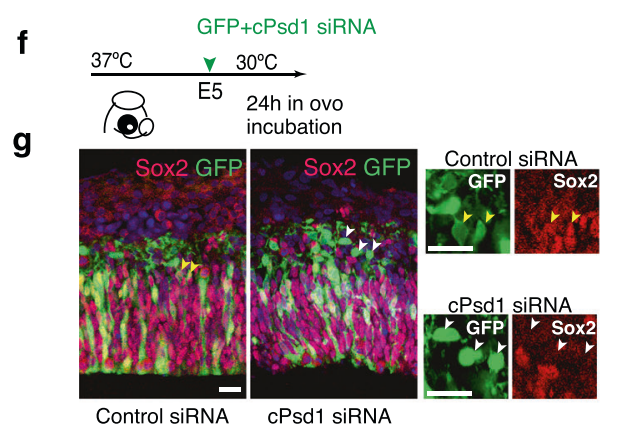

f

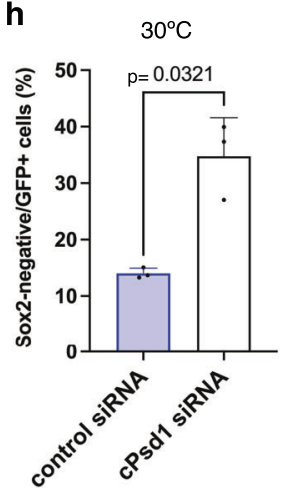

Fig. 4 PE mediates hypothermia-dependent increases in Notch activity. a Trapping of cell surface PE in cultured chick neural progenitors by treatment with SA-Ro for $12 \mathrm{~h}$. b Notch reporter activity in chick neural progenitors treated with SA-Ro at different temperatures (mean $+\mathrm{SD}, n=3$ biologically independent samples in each group). c Western blotting of Myc-tagged chick Psd1 in HEK293T cells transfected with siRNAs. d Introduction of the Notch reporter vector and siRNA targeting Psd1 in isolated chick neural progenitors. e Notch reporter activity in chick neural progenitors transfected with control or Psd1 siRNAs (mean + SD, $n=3$ biologically independent samples in each group). $\mathbf{f}$ Schematic illustration of cPsd1 siRNA introduction in the developing chick pallium. $\mathbf{g}$ Distributions of GFP-positive cells in control and cPsd1 siRNA overexpressing chick pallium incubated at $30^{\circ} \mathrm{C}$. $\mathbf{h}$ The proportion of Sox2negative and GFP-positive cells in control and cPsd1 siRNA overexpressing chick pallium at $30^{\circ} \mathrm{C}$ (means $+\mathrm{SD}, n=3$ biologically independent samples in each group). Ordinary one-way ANOVA for $\mathbf{b}$ ( $p$-values were adjusted by Tukey test for multiple comparisons), two-sided, unpaired $t$-test for $\mathbf{e}$, $\mathbf{h}$. $P$-values are indicated in each graph. Scale bars: $25 \mu \mathrm{m}$.

Enrichment of PE affects Notch signal in chick progenitors. To examine the functional contribution of PE to Notch signaling, we treated chick neural progenitors with SA-Ro for $12 \mathrm{~h}$ to trap cell surface PE and examined Notch reporter activity (Fig. 4a). SA-Ro application significantly reduced Notch activity in neural progenitors under both normothermic and hypothermic conditions (Fig. 4b). To further confirm the role of PE in Notch signaling, we designed an siRNA that downregulates $P s d 1$, a gene responsible for PE synthesis. The knockdown of chick Psd1 using siRNA significantly decreased Notch reporter activity in chick neural progenitors under hypothermic conditions (Fig. 4c-e). These results indicate that hypothermia-dependent activation of Notch signaling is mediated by increased PE synthesis and cell surface exposure in chick neural progenitors. To determine whether altered PE synthesis affects neuronal differentiation, we introduced siRNA targeting chick $P s d 1$ together with a GFP expression vector into the developing chick pallium. Compared to the results in the controls, the electroporation of Psd1 siRNA significantly increased the proportion of Sox2-negative cells delaminated from the ventricular zone (Fig. $4 \mathrm{f}-\mathrm{h}$ ), suggesting that reduced $\mathrm{PE}$ synthesis in ventricular progenitors increased neuronal differentiation. Taken together, these data indicate that temperature- dependent changes in PE synthesis play a significant role in the regulation of neurogenesis by modifying Notch signaling in the developing chick brain.

Notch signal is stable to temperature shifts in mouse. To further explore the evolutionary conservation of temperaturedependent neurogenic controls among amniotes, we investigated the thermal sensitivity of neurogenesis in the developing mammalian neocortex. Thus, we isolated mouse E14.5 brains and performed organotypic culture under normothermic $\left(37^{\circ} \mathrm{C}\right)$ and hypothermic $\left(30^{\circ} \mathrm{C}\right)$ conditions for $24 \mathrm{~h}$ (Fig. 5a). Neocortical neural progenitors were labeled by administration of EdU or electroporation of a GFP expression vector prior to the organotypic culture. At $24 \mathrm{~h}$ after the temperature shift, we examined cell cycle re-entry and neuronal differentiation in the neocortex cultured under different temperature conditions. Notably, the proportions of Ki67-positive or Sox2-negative cells in the progeny of labeled progenitors were comparable between normothermic and hypothermic conditions (Fig. $5 \mathrm{~b}-\mathrm{f}$ ), suggesting that hypothermia did not alter the rate of cell cycle re-entry and neuronal differentiation in the cultured mouse neocortex. Furthermore, cultured neural progenitors isolated from the neocortex exhibited stable 
a

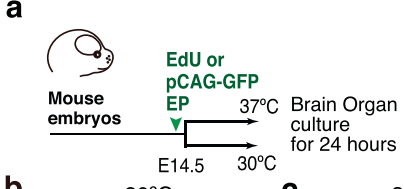

b
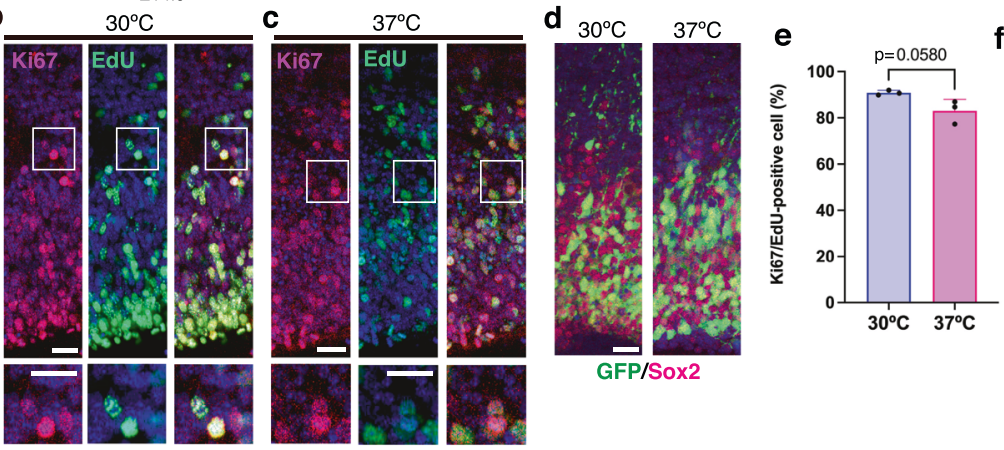

GFP/Sox2

g

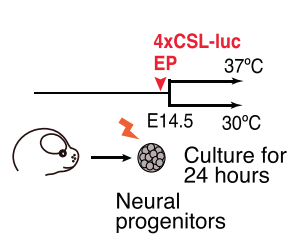

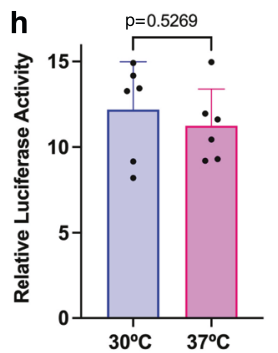
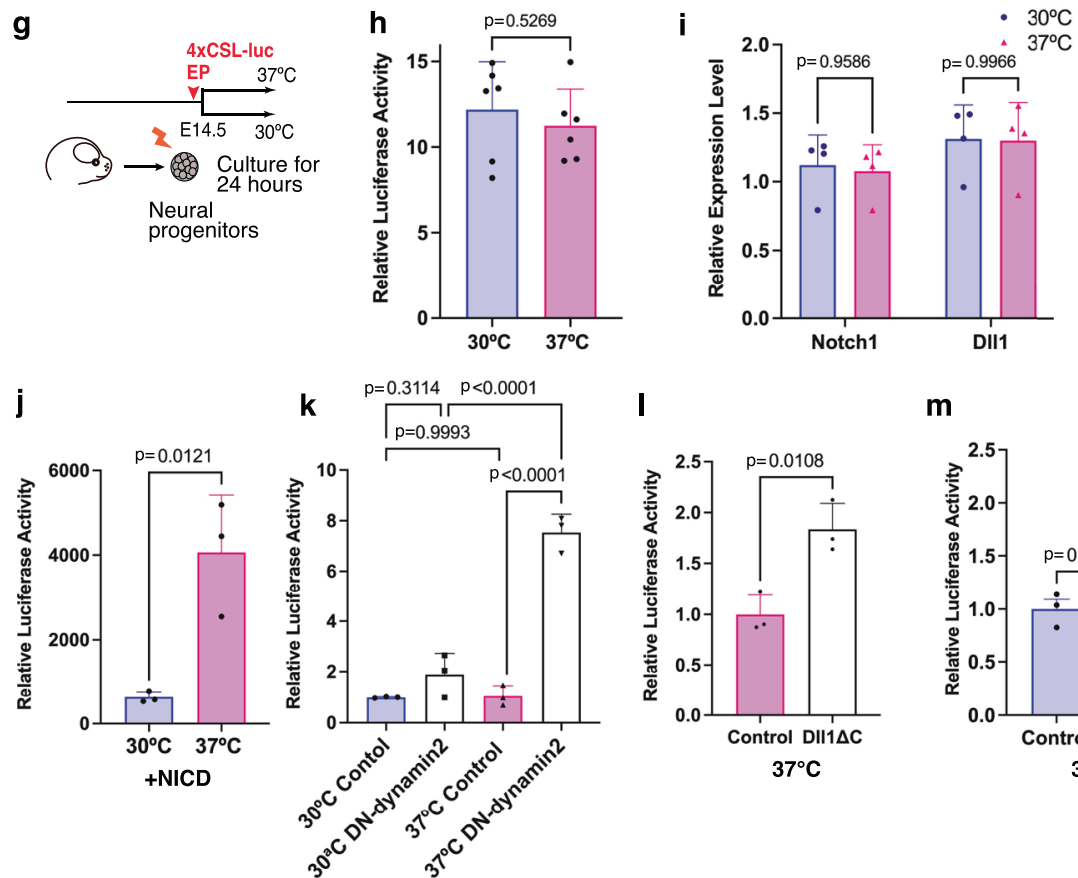

I

m
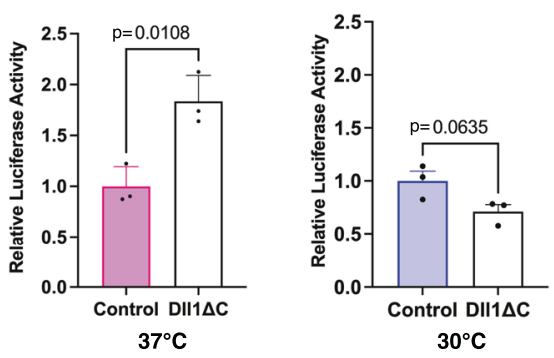

Fig. 5 Temperature robustness of Notch signaling in mouse neural progenitors. a Schematic illustration of the temperature shift experiment conducted on the developing mouse brain. b, c Distribution of Ki67-positive and/or EdU-positive cells in the developing mouse neocortex cultured at $30{ }^{\circ} \mathrm{C}$ (b) or $37^{\circ} \mathrm{C}$ (c). d Distribution of GFP-positive cells in the developing mouse neocortex cultured at $30^{\circ} \mathrm{C}$ or $37^{\circ} \mathrm{C}$. e, $\mathbf{f}$ The proportion of Ki67/EdU-positive cells (e) and Sox2-negative/GFP-positive cells (f) in the developing mouse neocortex cultured at $30^{\circ} \mathrm{C}$ or $37^{\circ} \mathrm{C}$ ( $m e a n+\mathrm{SD}, n=3$ biologically independent samples in each group). $\mathbf{g}$ Monitoring of Notch activity in mouse neocortical neural progenitors cultured at $30^{\circ} \mathrm{C}$ or $37^{\circ} \mathrm{C}$ for $24 \mathrm{~h}$. $\mathbf{h}$ Notch reporter activities in mouse neural progenitors cultured at $30^{\circ} \mathrm{C}$ or $37^{\circ} \mathrm{C}$ (means $+\mathrm{SD}, n=6$ biologically independent samples in each group). i Relative expression levels of genes encoding Notch1 and DII1 in mouse neural progenitors cultured at $30^{\circ} \mathrm{C}$ or $37^{\circ} \mathrm{C}$ (means $+\mathrm{SD}, n=4$ biologically independent samples in each group). j NICD-dependent Notch reporter activities in mouse neural progenitors cultured at $30^{\circ} \mathrm{C}$ or $37^{\circ} \mathrm{C}$ (means $+\mathrm{SD}, n=3$ biologically independent samples in each group). $\mathbf{k}$ Notch reporter activities in control and dominant-negative dynamin 2 (DN-dynamin 2)-overexpressing neural progenitors cultured at $30^{\circ} \mathrm{C}$ or $37^{\circ} \mathrm{C}$ (means $+\mathrm{SD}, n=3$ biologically independent samples in each group). $\mathbf{I}$, $\mathbf{m}$ Notch reporter activity in control neural progenitors and neural progenitors overexpressing DII1 lacking intracellular domain (DII1 $\Delta \mathrm{C}$ ) cultured at $37^{\circ} \mathrm{C}$ or $30^{\circ} \mathrm{C}$ (mean $+\mathrm{SD}, n=3$ biologically independent samples in each group). Two-sided, unpaired $t$-test $(\mathbf{e}, \mathbf{f}, \mathbf{h}, \mathbf{j}, \mathbf{l}, \mathbf{m}$ ), ordinary one-way or two-way ANOVA for $\mathbf{k}$ and $\mathbf{i}$ ( $p$-values were adjusted by Tukey or Sidak test for multiple comparisons). Scale bars: $20 \mu \mathrm{m}$.

Notch activity irrespective of the temperature (Fig. $5 \mathrm{~g}, \mathrm{~h}$ ). Accordingly, the expression levels of Notch1 and Dll1 in mouse neural progenitors did not differ between normothermic and hypothermic conditions (Fig. 5i). These data indicate that Notch signaling in the developing mouse neocortex is robust to a range of temperatures. To investigate potential mechanisms for stabilizing Notch activity under different temperatures, we introduced several constructs that modulate Notch signaling and compared the outcomes under normothermic and hypothermic conditions. We confirmed that NICD-dependent Notch activity was temperature-sensitive and was increased by temperature (Fig. 5j). Next, we investigated the temperature sensitivity of liganddependent endocytic pathways in mouse neocortical progenitors. The introduction of DN-dynamin 2 significantly increased Notch activity at $37^{\circ} \mathrm{C}$, suggesting that endocytosis negatively regulates Notch signaling in mouse neural progenitors, in contrast to the 
finding in chick neural progenitors. (Fig. 5k). Notch activity was also increased by Dll $\Delta \mathrm{C}$ in mouse neural progenitors at $37^{\circ} \mathrm{C}$ (Fig. 5l), corroborating that ligand-mediated endocytosis negatively controls Notch signaling. Notably, the introduction of DNdynamin 2 or Dll $1 \Delta \mathrm{C}$ did not facilitate Notch activity under hypothermic conditions (Fig. 5k, m), indicating that the endocytosis-dependent downregulation of Notch activity is also temperature-sensitive. These data suggest that the temperaturedependent transcriptional regulation of Notch signaling is counterbalanced by endocytosis-mediated negative regulation. In contrast to chick neural progenitors, we could not detect notable differences in phospholipid contents in mouse neural progenitors between normothermic and hypothermic conditions (Fig. S5a, b). Accordingly, neither the up- nor downregulation of Psd1 altered neuronal differentiation in the developing mouse neocortex (Fig. S5c-f). Thus, the thermal robustness of Notch signaling is not associated with temperature-dependent lipid synthesis in developing mouse neocortical progenitors.

Species-specific developmental responses to Notch alteration. In contrast to oviparous embryos, which are influenced by ambient temperatures, mammalian embryogenesis proceeds within the uterus under stable thermal conditions, raising the question of the biological significance of the thermal robustness of Notch signaling in the developing mammalian brain. One possible interpretation is that the species-specific thermal dependency of Notch signaling is associated with differential susceptibility of developmental programs to altered Notch signaling. We have previously identified species differences in pallial neurogenic patterns and the regulation of developmental signaling in amniotes ${ }^{34-37}$. Mammalian corticogenesis proceeds in an inside-out manner, in which excitatory neurons born in the ventricular zone radially migrate toward the surface of the cortex and adopt a position in a specific cortical layer. In contrast, reptilian and avian palliogenesis proceed in a roughly outside-in manner, in which newly born neurons do not undergo extensive radial migration but intermingle with previously born neurons. Thus, it is possible that stabilizing Notch signaling in mammalian neocortical development is necessary to avoid developmental abnormalities caused by Notch signaling perturbation. To test this possibility, we overexpressed an NICD expression vector in different amniote species (mice, chickens, and turtles) and examined long-term effects on neuronal distribution after electroporation. We labeled neural progenitors when massive neurogenesis occurs in the pallium depending on higher Notch activity, then fixed the embryo at the end of neurogenesis in each species ${ }^{34}$. Accordingly, we electroporated the NICD expression vector into the E14.5 mouse neocortex (Fig. 6a). As previously reported ${ }^{38,39}$, NICD overexpression suppressed neuronal differentiation in the developing mouse neocortex, which resulted in the delay of radial migration and the accumulation of transfected cells in the ventricular and subventricular zones (Fig. 6b,c). Abnormal cell accumulation in the subventricular zone was not fully restored in the postnatal mouse neocortex (Fig. S6a-c), indicating that the effect of altered Notch signaling was not fully compensated. Next, we addressed the effect of the transient activation of Notch signaling in the developing chicken pallium. To precisely compare homologous structures of the neocortex in the chick pallium, we targeted the dorsal pallium of early embryonic stages (E3.5, $\mathrm{HH} 20-22)$ and examined the cellular distribution at the end of neurogenesis (E10 or E11, Fig. 6d). The electroporation of the NICD expression vector significantly increased the activity of the Hes1 promoter in the developing chick pallium (Fig. S6d-k), corroborating that Notch-dependent downstream regulation is shared among amniotes. In contrast to the finding in the mouse neocortex, however, we could not detect obvious alterations in the cell distribution in the chick pallium after transient Notch activation (Fig. 6e, f). In both controls and NICD transfected embryos, GFP-positive cells were dispersed from deep to superficial parts of the chick dorsal pallium (Fig. 6f), indicating that the effect of altered Notch signaling was restored in the developing chick pallium. Similarly, the siRNA-mediated knockdown of Psd1 did not affect the cell distribution in the developing chick pallium (Fig. S61-n). We also confirmed that the neuronal distribution in the developing turtle pallium was not affected by transient NICD overexpression (Fig. S6o-r). These results suggest that mammalian neocortical development is vulnerable to the transient perturbation of Notch signaling, while the developmental programs of the reptilian and avian pallium retain the buffering potential to stabilize phenotypes in response to Notch-dependent changes during embryogenesis. Thus, the thermal robustness of Notch signaling in mammalian neural progenitors might provide a developmental safeguard to prevent deleterious phenotypic consequences in the developing mammalian neocortex.

\section{Discussion}

The species-specific thermal sensitivity of developmental signaling plays a crucial role in the establishment of phenotypic plasticity and robustness in response to temperature variations. Here, we report temperature-dependent changes in neurogenesis in developing reptilian and avian brains, which are mediated by the thermal sensitivity of Notch signaling. Intriguingly, Notch activity is increased by a lower temperature $\left(30^{\circ} \mathrm{C}\right)$, corresponding to hypothermic conditions for chick embryos but normothermia for turtles, indicating that the thermal responsiveness of Notch signaling is shared among oviparous amniotes regardless of species differences in optimal temperatures for embryogenesis. In contrast, Notch activity in developing mouse neural progenitors is highly stable to temperature variations, indicating that differential regulatory mechanisms underlie temperature-dependent regulation of Notch activity between oviparous and viviparous amniotes, despite the extensive conservation of Notch signaling components among species. Increased levels of NICD in the developing chick pallium reduced cell cycle re-entry, corroborating that the hypothermia-dependent inhibition of cell cycle progression is mediated by elevated Notch signaling, as previously reported $^{40,41}$. It is possible that S-phase, during which DNA synthesis occurs, is more sensitive to temperature changes than the mitotic phase in developing neural progenitors.

We found that dynamin-dependent ligand endocytosis is critical for the hypothermia-dependent activation of Notch signaling in developing chick neural progenitors. Several studies have shown that the ubiquitylation and internalization of Notch ligands play a key role in Notch signaling, by producing a "pulling force" that promotes receptor cleavage and/or promotes the recycling of ligands on the cell surface ${ }^{42}$. Accordingly, the lower temperature increased endogenous ligand-dependent Notch activation in chick neural progenitors, suggesting that endocytosis positively regulates hypothermia-induced Notch activation by facilitating the receptor-ligand interaction between juxtaposed cells, or the recycling of ligands in cells sending out signals. In Drosophila, lower temperatures reduce ligand-dependent Notch activation, which is compensated by ligand-independent endocytic pathways ${ }^{18}$. In contrast to the finding in chicks, we found that endocytosis negatively regulates Notch signaling in developing mouse neural progenitors, possibly by facilitating degradation pathways of Notch components. Thus, the divergent functions of endocytosis in Notch signaling underlie the speciesspecific thermal sensitivity of Notch signaling, although further investigations are required to reveal the detailed molecular 
a
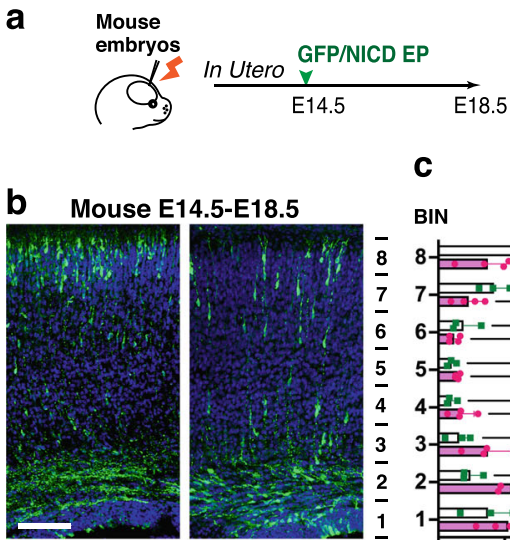

Control

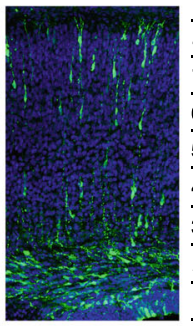

NICD
C

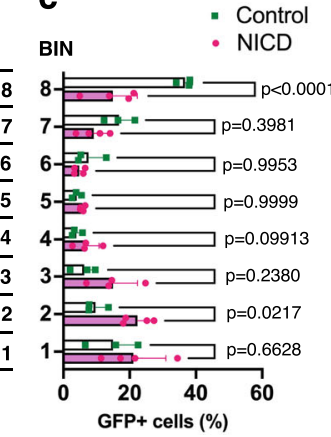

$$
\text { GFP+ cells (\%) }
$$

d
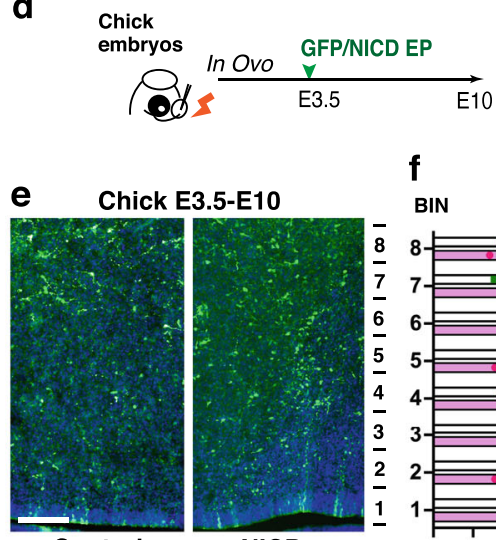

Control

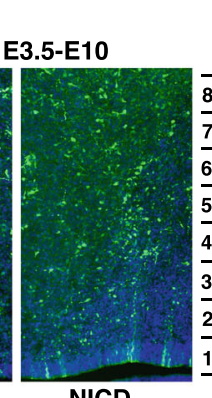

NICD

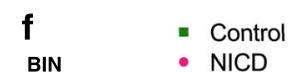

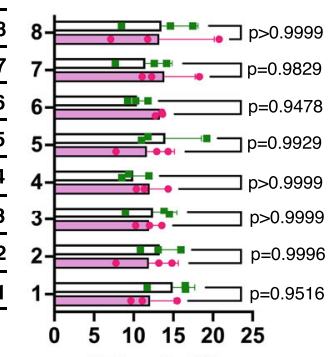

GFP+ cells (\%)

g

Mouse neocortex

\begin{tabular}{lll}
\hline $\begin{array}{l}\text { Temperature responsiveness of } \\
\text { Notch activity }\left(30^{\circ} \mathrm{C} \text { vs } 37^{\circ} \mathrm{C}\right)\end{array}$ & Stable to temperature change & Increased by hypothermia \\
\hline $\begin{array}{l}\text { Temperature responsiveness of } \\
\text { PE synthesis }\left(30^{\circ} \mathrm{C} \text { vs } 37^{\circ} \mathrm{C}\right)\end{array}$ & Stable to temperature change & Increased by hypothermia \\
\hline $\begin{array}{c}\text { Effects of increased Notch } \\
\text { signaling (short-term) }\end{array}$ & Reduced neuronal differentiation & Reduced neuronal differentiation \\
\hline $\begin{array}{c}\text { Effects of increased Notch } \\
\text { signaling (long-term) }\end{array}$ & Delay of radial migration & Mammals (Theria)
\end{tabular}

Fig. 6 Species-specific susceptibility to altered Notch signaling in amniote pallium. a-f Transient NICD overexpression in the developing mouse neocortex (a-c) and chick pallium (d-f) by in utero or in ovo electroporation. a Schematic illustration of in utero electroporation. b, c Distribution of GFPpositive neurons in the developing mouse neocortex electroporated with the control or NICD expression vector (mean $+\mathrm{SD}, n=3$ biologically independent samples in each group). d Schematic illustration of the in ovo electroporation experiment. e, $\mathbf{f}$ Distribution of GFP-positive neurons in the developing chick dorsal pallium electroporated with control or NICD expression vector (mean $+S D, n=3$ biologically independent samples in each group). $\mathbf{g}$ Summary of the species-specific temperature responsiveness of Notch signaling, PE synthesis, and the effects of increased Notch signaling in the developing amniote pallium ('Refs. ${ }^{16,38}$ ). Two-way ANOVA for $\mathbf{c}, \mathbf{f}$ ( $p$-values were adjusted by Tukey test). Scale bars: $100 \mu \mathrm{m}$.

mechanisms counterbalancing the positive and negative regulation of Notch activation in response to temperature.

We found that a hypothermia-dependent increase in PE contributes to increased Notch activity in developing chick neural progenitors. $\mathrm{PE}$ is a major glycerophospholipid that is enriched in the inner leaflet of plasma membranes and significantly affects the fluidity of lipid bilayers, as well as the topology of membraneassociated proteins ${ }^{29,43}$. Furthermore, the exposure of PE on the cell surface contributes to specific cellular events, such as cell mitosis $^{32}$ or cell fusion ${ }^{33}$. We speculate that the enrichment of PE at the cell surface facilitates Notch signaling at multiple levels, including changes in the distribution and conformation of Notch receptors and/or ligands, as well as ligand internalization and recycling to the plasma membrane. A recent study reported that the N-terminus of Notch ligands functions as a phospholipid recognition domain and mediates Notch activation ${ }^{44}$, also suggesting that excess cell surface $\mathrm{PE}$ promotes Notch signaling by facilitating ligand-dependent cell-cell interactions under hypothermic conditions.

Although temperature-dependent Notch activation is shared among oviparous amniote species, the profound increase in $\mathrm{PE}$ synthesis observed in developing chick neural progenitors is unique to these cells, implying that the temperature sensitivity of
Notch signaling is mediated by variable regulatory mechanisms depending on the species-specific cellular metabolism. We found that the major molecular species of chicken PE are composed of monounsaturated fatty acids, while mouse and turtle PE contain polyunsaturated fatty acids. The former can be produced by de novo synthesis through intracellular metabolic pathways, but the latter is derived from external supplements ${ }^{45,46}$. Thus, speciesspecific differences in lipid metabolism are a key factor conferring phenotypic plasticity and robustness in variable environments, in addition to underlying cold acclimation ${ }^{47}$ and longevity ${ }^{48,49}$, as a result of adaptive evolution in a variety of species.

The transient overexpression of Notch signaling components results in long-term impacts on the cell distribution in the mammalian neocortex but not in the pallium of nonmammalian amniotes. We have previously shown species-specific patterns of neuronal migration in the developing amniote pallium, among which the sequential production of excitatory neuron subtypes and the locomotive mode of neuronal migration are unique characteristics of the developing mammalian neocortex ${ }^{37}$. It has also been reported that NICD controls the radial migration of cortical neurons downstream of Reelin signaling ${ }^{39}$, which is greatly amplified in the developing mammalian neocortex ${ }^{50}$. Thus, we suggest that the temperature robustness of Notch 
signaling is crucial for canalizing cytoarchitectonic phenotypes minimizing developmental abnormalities in the mammalian neocortex. In contrast, pallial development in reptiles and birds exhibits phenotypic robustness to the acceleration or deceleration of neuronal differentiation depending on Notch signaling, which might underlie the evolutionary adaptation of oviparous amniotes in response to variable environmental temperatures (Fig. 6g). Indeed, temperature fluctuations during embryogenesis are common in many ectothermic animals, and are inversely correlated with the total length of the developmental period depending on the variable climate ${ }^{51}$. Conversely, temperature perturbations also increase the risk of embryonic mortality and developmental malformation in both oviparous and viviparous animals ${ }^{6}$. We found that hypothermia induced global changes in transcriptome components related to a wide range of cellular events, raising further questions regarding the physiological significance of other metabolic pathways in temperature-dependent phenotypic variations, which is a critical issue that remains to be elucidated in future studies.

\section{Methods}

Animals. Fertilized chicken eggs (Gallus gallus) were obtained from a local farm (Yamagishi, Japan) and incubated at $37.0 \pm 0.2^{\circ} \mathrm{C}$ until the temperature shift. Embryonic stages were determined according to Hamburger-Hamilton stages ${ }^{52}$. At E5 (HH stages 24-25), a group of eggs was transferred to $30.0 \pm 0.2^{\circ} \mathrm{C}$ and incubated for $24 \mathrm{~h}$. Fertilized eggs of Chinese softshell turtles (Pelodiscus sinensis) were obtained from a local farm (Daiwa Yoshoku, Japan) and incubated at $28 \pm 0.5^{\circ} \mathrm{C}$. The embryonic stages of the turtles were determined according to developmental stages provided in a previous report ${ }^{53}$. Pregnant female mice [Mus musculus, Crl:CD-1 (ICR), 3 months] were obtained from Japan SLC, Inc. The day on which vaginal plugs were found at midday was considered embryonic Day 0.5 (E0.5). Total 17 pregnant mice were used in this study. Diets for mice (MF, Oriental Yeast Co., Ltd.) were purchased from Shimizu Laboratories Supplies Co., Ltd. Housing conditions of mice were as follows: $12 \mathrm{~h}$ dark/light cycle, $25^{\circ} \mathrm{C}$, and $60 \%$ humidity. All experimental procedures conducted in this study were approved by the experimental animal committee of Kyoto Prefectural University of Medicine and Institutional Animal Care and they were performed in accordance with the relevant guidelines (M2020-193, M2020-4).

EdU pulse-labeling. The EdU labeling of neurons in the chick pallium and mouse neocortex was performed according to a previous study ${ }^{34}$. Briefly, $\sim 0.05 \mu \mathrm{L}$ of EdU (Thermo Fisher Scientific, $10 \mathrm{mg} \mathrm{mL}^{-1}$ ) was injected into the lateral ventricle of each chick embryo (HH stages $24-25$ ), and the eggs were incubated at $30^{\circ} \mathrm{C}$ or $37^{\circ} \mathrm{C}$ for $24 \mathrm{~h}$. To examine EdU-labeled cells were detected with the Click-iT EdU Detection System (Thermo Fisher Scientific).

Plasmids. The following expression vectors were used in the present study: p4xCSL-firefly luciferase (Addgene \#41726), pCAGGS-NICD (Addgene \#26891), DN-dynamin 1 (Dynamin 1 K44A, a gift from Dr. Sandra Schmid), DN-dynamin 2 (GFP-dynamin 2 K44A, Addgene \#22301), HA-Ubiquitin (Addgene \#18712), pHR_SFFV_LaG17_synNotch_TetRVP64 (Addgene \#79128), pHR_EGFPligand (Addgene \#79129), pCS2-Notch1 FL-6MT (Addgene \#41728), pCMV-mouse Dll1 (OriGene), pCMV-mouse Dll4 (OriGene), p6872 pHAGE-N-V5-MAML1-FL (Addgene \#37048), and pHes1-GFPd2 (Addgene \#14808). pCS2-Notch1 L468A was constructed by site-directed mutagenesis using the PrimeSTAR Mutagenesis Basal Kit (Takara). DN-MAML1 lacking the NICD-binding domain (13L-74H) and Dll1 $\Delta \mathrm{C}$ and Dll4 $\Delta \mathrm{C}$ lacking the intracellular domain (Dll1:569V-728L, Dll4:552A-686V) were amplified by polymerase chain reaction (PCR) and subcloned into pCAG-RB using the In-Fusion HD Cloning Kit (Clontech). pCMVHA-Notch1 and pCMV-Myc-Ubiquitin were constructed by subcloning the cDNAs of Notch1 or Ubiquitin into pCMV-HA-N or pCMV-Myc-N (Clontech), respectively. Mouse and chick Psd1 cDNAs (ENSMUSG00000023452 and ENSGALT00000011126.6, respectively) were chemically synthesized and subcloned into the pGL3-promoter or pCMV-Myc-N vector.

Luciferase reporter assay. To quantify Notch signaling activity, p4xCSL-firefly luciferase and pRL-SV40 vectors (Promega) were coelectroporated into dissociated neural progenitors using electroporation cuvettes (SE-202, BEX) and a pulse generator (CUY21-EDIT, BEX). After electroporation, the neural progenitors were cultured as floating cell aggregates in a neurobasal medium supplemented with GlutaMAX, the B27 supplement (Thermo Fisher Scientific) and FGF2 (Thermo Fisher Scientific, $10 \mathrm{ng} / \mathrm{mL}$ ), as previously described ${ }^{54}$. Chicken primary liver cells and heart muscle cells were isolated from E10 chick embryos and dissociated with $0.25 \%$ trypsin. Chicken fibroblast cells (DF-1) were purchased from the ATCC (CRL-12203). These cells were cultured at $30 \pm 0.2^{\circ} \mathrm{C}$ or $37 \pm 0.2^{\circ} \mathrm{C}$ for $24 \mathrm{~h}$ in incubators supplied with $5 \% \mathrm{CO}_{2}$. To monitor Notch activity in these cell types, p4xCSL-firefly and pRL-SV40 were transfected into the cells by using Lipofectamine 2000 (Thermo Fisher Scientific), and the cells were cultured in Dulbecco's modified Eagle's medium (DMEM) supplemented with fetal bovine serum. To block $\gamma$-secretase activity or dynamin-dependent endocytosis, DAPT (Sigma, $10 \mu \mathrm{M}$ ) or Dynasore (Abcam, $50 \mu \mathrm{M}$ ) was added to the culture medium for $24 \mathrm{~h}$. Luciferase activity was examined with the Dual-Luciferase Reporter Assay System (Promega). Chemical luminescence was analyzed with a luminometer (GENE LIGHT GL210A, Microtec). All firefly luciferase values were normalized to Renilla luciferase activities to quantify relative luciferase units. To detect firefly luciferase activity driven by the SynNotch system, HEK293T cells were purchased from RIKEN Bioresource Center (RBRC-RCB2202) and transfected with

pHR_SFFV_LaG17_synNotch_TetRVP64 and cultured alone or mixed with neural progenitors transfected with pHR_EGFPligand. Each experiment was carried out with at least three biologically independent samples.

Immunohistochemistry. Brains were fixed with $4 \%$ paraformaldehyde dissolved in phosphate-buffered saline (PBS) at $4{ }^{\circ} \mathrm{C}$ overnight. After washing with PBS, the brains were cryoprotected with a $30 \%$ sucrose solution and immersed in TissueTek. The frozen brains were sectioned at a thickness of $20 \mu \mathrm{m}$ using a Cryostat (Leica CM1850, Germany), and incubated with primary antibodies, including antiSox2 (Abcam, ab97959, rabbit polyclonal,1:1000), anti-phosphorylated histone H3 (Merck, 05-806, rabbit polyclonal, 1:1000), anti-GFP (Nakalai Tesque, 04404-84, rat monoclonal, 1:1000), and anti-Ki67 (Abcam, ab15589, mouse monoclonal, 1:1000) antibodies. After washing, the sections were incubated with secondary antibodies, including Alexa-Fluor 488, 594 or 633-conjugated anti-rabbit and antirat antibodies (Life Technologies, 1:500). Fluorescent images were captured with fluorescent microscopes (SZX7, OLYMPUS; BX51, OLYMPUS) equipped with a cooled CCD camera (DP80, OLYMPUS) and a laser confocal microscope (FV1000D, Olympus). All captured images were processed with cell Sense standard (v.1.17, OLYMPUS), FV10-ASW (v4.2, OLYMPUS), ImageJ (Image analysis in Java 1. $45 \mathrm{~s}, \mathrm{NIH}$ ), and Adobe Photoshop (v22.42, Adobe Systems).

Coimmunoprecipitation and western blotting. Cultured chick neural progenitors transfected with pCMV-Dll1-HA and pCMV-ubiquitin-Myc were lysed in RIPA buffer (20 mM Tris, 150 mM NaCl, 1 mM EDTA, 1\% Nonidet P40, 1\% SDS, $0.1 \%$ deoxycholate, $1 \mathrm{mM} \mathrm{NaF}$ and protein inhibitor). Samples were incubated with antiHA agarose (Pierce HA Tag IP/Co-IP application set, Thermo Fisher Scientific) at $4{ }^{\circ} \mathrm{C}$ overnight. For western blotting, proteins were transferred to PVDF membranes, which were then incubated with anti-c Myc (MBL, 562, rabbit polyclonal, 1:1000) or anti-HA (BioLegend, MMS-101R, mouse monoclonal, 1:1000), followed by HRP-conjugated anti-mouse or anti-rabbit IgG (Vector Laboratories, USA), developed with Chemi-Lumi One Super (Nacalai Tesque, Japan) and analyzed with a luminescent image analyzer (LAS-2000, Fujifilm, Japan). To detect NICD and Dll1 by western blotting, an anti-NICD (Merck, 07-1232, rabbit polyclonal, 1:1000), and ant-Dll1 antibody (R\&D systems, AF5026, sheep polyclonal, 1:1000) was used as the primary antibody. Anti- $\alpha$-tubulin antibody (Abcam, ab9267, rat monoclonal, 1:1000) and $\beta$-actin antibody (Abcam, ab8277, rabbit polyclonal, $1: 1000)$ were used as the primary antibody for the internal control of western blotting.

Whole-brain culture. The whole-brain culture was performed following a modified version of a protocol form a previous report ${ }^{54}$. Isolated mouse brains were cultured in rotating culture bottles filled with DMEM supplemented with fetal bovine serum (10\%) and antibiotics (penicillin/streptomycin). The whole-brain culture was performed by using a whole-embryo culture system (IKEMOTO Sci Inc.) supplied with $60 \% \mathrm{O}_{2}$ at $30^{\circ} \mathrm{C}$ or $37^{\circ} \mathrm{C}$ for $24 \mathrm{~h}$.

In ovo electroporation. The in ovo electroporation of the developing chick and turtle pallium was performed according to a previous study 34,55 . Briefly, a small window was opened in the shell of an incubated egg, and a small amount of DNA solution (less than $0.05 \mu \mathrm{L}$ ) was injected into the lateral ventricle with a small glass needle. Needle-type electrodes (CUY200S, BEX) were placed on the embryonic head, and square electric pulses ( $28 \mathrm{~V}$ for $50 \mathrm{~ms}, 2$ or 3 times) were applied to target the pallium with a pulse generator (CUY21 EDITII, BEX). After electroporation, the extraembryonic cavity was filled with sterilized Hank's balanced salt solution (HBSS) containing antibiotics (penicillin/streptomycin and gentamycin), and the window was sealed. The electroporated embryos were incubated in an incubator at $30^{\circ} \mathrm{C}$ or $37^{\circ} \mathrm{C}$ for $24 \mathrm{~h}$. To examine S-phase re-entry in the developing chick pallium, we first electroporated the GFP expression vector in the pallium, by which proliferating ventricular progenitors were efficiently labeled ${ }^{56}$, then we administrated the EdU solution into the lateral ventricle $2 \mathrm{~h}$ before fixation.

In utero electroporation. In utero electroporation was performed according to previously described ${ }^{57}$. Briefly, a pregnant mouse was anesthetized with isoflurane, and an incision was made in the abdominal wall to expose the uterine horns. A small amount of DNA solution $(0.3-0.5 \mu \mathrm{L})$ was injected into the lateral ventricle of each embryo, and square pulses ( $35 \mathrm{~V}, 50 \mathrm{~ms}$, four times) were applied to the 
embryos with a tweezer-type electrode (CUY650P3, BEX) connected to a pulse generator (BEX).

RNA-sequencing data analysis. Total RNA was extracted from cultured chick neural progenitors according to the manufacturer's protocols (Novogene). RNA quality was assessed using a NanoPhotometer spectrophotometer (IMPLEN, USA). The cDNA library was constructed using a NEBNext Ultra RNA Library Prep Kit for Illumina and sequenced on an Illumina HiSeq platform and $125 \mathrm{bp} / 15 \mathrm{bp}$ paired-end reads were generated. The sequence data were mapped to a reference genome sequence [Galgal4: https://www.ncbi.nlm.nih.gov/assembly/ GCF_000002315.3/).

Analysis of phospholipid compositions. Total lipids were extracted from a cultured chick, mouse, and turtle neural progenitors using the Bligh \& Dyer method ${ }^{58}$ and dissolved in chloroform. Phospholipid contents were determined by inorganic phosphate quantification ${ }^{59}$. The analysis of phospholipids was performed with a high-performance liquid chromatography system (LC-30AD, Shimadzu, Kyoto, Japan) coupled to a triple quadrupole mass spectrometer (LC-MS-8040, Shimadzu) equipped with an electrospray source ${ }^{60}$. Separation was performed in a Kinetex C8 column $(2.6 \mu \mathrm{m}, 2.1 \times 150 \mathrm{~mm})$ (Phenomenex, Torrance, CA, USA) with a binary mobile phase with the following composition: $10 \mathrm{mM}$ ammonium formate in water (mobile phase A) and $10 \mathrm{mM}$ ammonium formate in 2-propanol/acetonitrile/water $(45: 45: 10, \mathrm{v} / \mathrm{v} / \mathrm{v})$ (mobile phase B). The pump controlling the gradient of mobile phase B was programmed as follows: an initial isocratic flow at $20 \%$ B for $1 \mathrm{~min}$, a linear increase to $40 \%$ B for $1 \mathrm{~min}$, an increase to $92.5 \%$ B using a curved gradient for $23 \mathrm{~min}$, a linear increase to $100 \%$ B for $1 \mathrm{~min}$, and hold at $100 \%$ B for $4 \mathrm{~min}$. The total flow rate was $0.3 \mathrm{ml} / \mathrm{min}$, the column temperature was $45^{\circ} \mathrm{C}$, and the sample temperature was $4{ }^{\circ} \mathrm{C}$. The spectrometer parameters were as follows: nebulizer gas flow $2 \mathrm{~L} / \mathrm{min}$, drying gas flow $15 \mathrm{~L} / \mathrm{min}$, interface voltage $4.5 \mathrm{kV}$, DL temperature $250^{\circ} \mathrm{C}$, and heat block temperature $400^{\circ} \mathrm{C}$. The multiple reaction monitoring transition was $[\mathrm{M}+\mathrm{H}]^{+} \rightarrow[184.1]^{+}$for $\mathrm{PC}$ and $[\mathrm{M}+\mathrm{H}]^{+} \rightarrow[\mathrm{M}+\mathrm{H}$ $-141.0]^{+}$for PE. The fatty acid composition of PC and PE was determined via the product ion scan analysis of $[\mathrm{M}+\mathrm{HCOO}]^{-}$and $[\mathrm{M}-\mathrm{H}]^{-}$as precursor ions, respectively. PC (12:0-13:0) and PE (12:0-13:0) (Avanti Polar Lipids, AL, USA) were used as internal standards.

Quantitative real-time PCR. Total RNA was extracted from cultured mouse neural progenitors with an RNeasy Mini Kit (Qiagen), and cDNA was synthesized using random primers with reverse transcriptase (ReverTra Ace, TOYOBO). qPCR was performed on a Light Cycler Nano (Roche, Switzerland) with THUNDERBIRD SYBR qPCR Mix (TOYOBO) according to the manufacturer's protocols. Gene expression levels were normalized to those of $\beta$-actin amplification. Primer sequences for $\mathrm{qPCR}$ were designed by primer-BLAST (NCBI). The primer sequences were as follows: 5'-ACGTAGTCCCACCTGCCTATG-3' and 5'ACAGGTGCCCTGATTGTAGCA-3' (mouse Notch1: ENSMUSG00000026923), 5'-CAACCAAGACCTGAACTACT-3' and 5'-CACTCATCTACTTCCAGCTC-3' (mouse Dll1: ENSMUG00000014773). qPCR was carried out on four biologically independent samples with two technical replicates.

Preparation of siRNAs. siRNAs that target mouse or chick Psd1 mRNA was designed with the publicly available software (siDirect v2.0) and chemically synthesized (BEX). The sense and antisense strand sequences of siRNAs were as follows: 5'-AUAAAUGAUUUUACUUUUCCA-3' and 5'-GAAAAGUAAAAUCAUUUAUAU-3' (mouse_Psd1 siRNA1), 5'-GAGUAUUUGGCCUAUAUUUAC3' and 5'- AAAUAUAGGCCAAAUACUCUA-3' (chick Psd1siRNA1), 5'GUAAAAGGGGUUACUUAUUCU-3' and 5'-AAUAAGUAACCCCUUUUACUU-3' (chick_Psd1siRNA4), 5'-GAAACAUGGCUUUUUCUCUUU-3' and 5'AGAGAAAAAGCCAUGUUUCCA-3' (chick_Psd1siRNA5), 5' CAAAUUCCACCUUAAAGCUGG-3' and 5'- AGCUUUAAGGUGGAAUUUGAA-3' (chick_Psd1siRNA6). A siRNA with no homologous sequences in eukaryotes was used as a negative control (Universal negative control siRNA, NEGS 210210, Nippon gene). To validate the knockdown efficiency, each siRNA was introduced into HEK293T cells together with the pGL3-promoter-mPsd1-3'UTR or pCMVchick Psd1-Myc vector and dual-luciferase assays or western blotting were performed. After $24 \mathrm{~h}$, chick Psd 1 protein was quantified by western blotting. The western blotting data of chick_Psd1siRNA1, 4, 5, and 6 are available from Source Data Files.

Incubation of chick neural progenitors with a PE-binding peptide. The application of a PE-binding peptide (SA-Ro) to cultured chick neural progenitors was performed as previously described ${ }^{32}$. Briefly, isolated chick neural progenitors were cultured on laminin-coated glass slides (Lab-Tek Chamber Slides 158599, Nunc) at $30^{\circ} \mathrm{C}$ or $37^{\circ} \mathrm{C}$ for $24 \mathrm{~h}$ and incubated with PBS containing $0.1 \%$ BSA followed by SA-Ro $\left(5 \mu \mathrm{g} \mathrm{mL}^{-1}\right)$ for $2 \mathrm{~h}$. After fixation with $4 \%$ PFA and extensive washing with PBS, chick neural progenitors were incubated with FITC-conjugated biotin (1:200) for $1 \mathrm{~h}$. To assess Notch activity by trapping cell surface PE, neurospheres transfected with p4xCSL-luc and pRL-SV40 were incubated with SA-Ro $\left(5 \mu \mathrm{g} \mathrm{mL}^{-1}\right)$ for $12 \mathrm{~h}$, and luciferase activity was examined.
Statistical analysis. For statistical analysis, three to six independent samples from each experimental group were compared. Comparisons between experimental groups were performed using Microsoft Excel (v16.54, Microsoft) and Prism 9 (v9.1.2, GraphPad Software Inc). All data are presented as the mean + SD. Statistical significance was determined using the two-tailed unpaired Student's $t$-test, ordinary one-way ANOVA with Tukey's multiple comparisons test, or two-way ANOVA with Sidak's multiple comparison test. For multiple comparisons, all $p$ values were adjusted. In the data obtained from Sox2-negative cells among GFPpositive cells, the matching of observed numbers and expected values was analyzed by using the chi-square test. Differential gene expression analyses of RNAsequencing data between the $30^{\circ} \mathrm{C}$ and $37^{\circ} \mathrm{C}$ conditions were performed using the DESeq R package (1.18.0, Bioconductor), which provides statistical routines for determining differential expression in digital gene expression data using a model based on the negative binomial distribution. The resulting $p$-values were adjusted using Benjamin-Hochberg's approach for controlling the false discovery rate. Gene Ontology (GO) enrichment analysis is performed with GOseq R package (Release 2.12, Bioconductor) based on the Wallenius non-central hypergeometric distribution for multiple comparisons. KEGG enrichment analysis based on the database https://www.genome.jp/kegg/ was performed using KOBAS software (v2.0, http://kobas.cbi.pku.edu.cn) and test the enrichment of differential expression genes in KEGG pathways. $P$-values were estimated by a hypergeometric test, and $p<0.05$ were considered statistically significant. $\mathrm{Q}$ value is the adjusted $p$ values after multiple hypothesis testing.

Reporting summary. Further information on research design is available in the Nature Research Reporting Summary linked to this article.

\section{Data availability}

The list of genes showing temperature-dependent expression in chick neural progenitors is available from Mendeley Data (https://doi.org/10.17632/9zxt47grif.2; https:// data.mendeley.com/datasets/9zxt47grif/1). The raw data of RNA sequencing derived from chick neural progenitors have been deposited to the DDBJ database (DRA012953). Sample information of RNA sequencing is also available on European Nucleotide Archive (https://www.ebi.ac.uk/ena/browser/view/DRA012953). All data generated in this study have been deposited to Mendeley Data (https://doi.org/10.17632/j2t6c343hg.1; https://data.mendeley.com/datasets/j2t6c343hg/1). Source data are provided with this paper.

Received: 18 October 2020; Accepted: 6 December 2021; Published online: 10 January 2022

\section{References}

1. Schmalhausen I. I. Factors of evolution: the theory of stabilizing selection. (The Blakiston Company, 1949).

2. Waddington, C. H. Selection of the genetic basis for an acquired character. Nature 169, 625-626 (1952).

3. Beldade, P., Koops, K. \& Brakefield, P. M. Developmental constraints versus flexibility in morphological evolution. Nature 416, 844-847 (2002).

4. Ehrenreich, I. M. \& Pfennig, D. W. Genetic assimilation: a review of its potential proximate causes and evolutionary consequences. Ann. Bot. 117, 769-779 (2016).

5. Gilbert S. F. Ecological developmental biology. (Sinauer Associates, 2015).

6. Hutson M. R. et al. Temperature-activated ion channels in neural crest cells confer maternal fever-associated birth defects. Sci Signal 10, eaa14055 (2017).

7. Irvine, S. Q. Embryonic canalization and its limits-A view from temperature. $J$ Exp. Zool. B Mol. Dev. Evol. 334, 128-144 (2020).

8. Kuntz, S. G. \& Eisen, M. B. Drosophila embryogenesis scales uniformly across temperature in developmentally diverse species. PLoS Genet. 10, e1004293 (2014).

9. Begasse, M. L., Leaver, M., Vazquez, F., Grill, S. W. \& Hyman, A. A. Temperature dependence of cell division timing accounts for a shift in the thermal limits of C. elegans and C. briggsae. Cell Rep. 10, 647-653 (2015).

10. Lucchetta, E. M., Lee, J. H., Fu, L. A., Patel, N. H. \& Ismagilov, R. F. Dynamics of Drosophila embryonic patterning network perturbed in space and time using microfluidics. Nature 434, 1134-1138 (2005).

11. Takeuchi, K. et al. Changes in temperature preferences and energy homeostasis in dystroglycan mutants. Science 323, 1740-1743 (2009).

12. Chen, J., Nolte, V. \& Schlotterer, C. Temperature stress mediates decanalization and dominance of gene expression in Drosophila melanogaster. PLoS Genet. 11, e1004883 (2015).

13. Kopan, R. \& Ilagan, M. X. The canonical Notch signaling pathway: unfolding the activation mechanism. Cell 137, 216-233 (2009).

14. Louvi, A. \& Artavanis-Tsakonas, S. Notch signalling in vertebrate neural development. Nat. Rev. Neurosci. 7, 93-102 (2006). 
15. Imayoshi, I., Sakamoto, M., Yamaguchi, M., Mori, K. \& Kageyama, R. Essential roles of Notch signaling in maintenance of neural stem cells in developing and adult brains. J. Neurosci. 30, 3489-3498 (2010).

16. Gaiano, N. \& Fishell, G. The role of notch in promoting glial and neural stem cell fates. Annu. Rev. Neurosci. 25, 471-490 (2002).

17. Mazaleyrat, S. L. et al. Down-regulation of Notch target gene expression by Suppressor of deltex. Dev. Biol. 255, 363-372 (2003).

18. Shimizu, H. et al. Compensatory flux changes within an endocytic trafficking network maintain thermal robustness of Notch signaling. Cell 157, 1160-1174 (2014).

19. McElhinny, A. S., Li, J. L. \& Wu, L. Mastermind-like transcriptional coactivators: emerging roles in regulating cross talk among multiple signaling pathways. Oncogene 27, 5138-5147 (2008)

20. Whiteman, P. et al. Molecular basis for Jagged-1/Serrate ligand recognition by the Notch receptor. J. Biol. Chem. 288, 7305-7312 (2013).

21. Morsut, L. et al. Engineering customized cell sensing and response behaviors using synthetic Notch receptors. Cell 164, 780-791 (2016).

22. Fortini, M. E. \& Bilder, D. Endocytic regulation of Notch signaling. Curr Opin. Genet Dev. 19, 323-328 (2009).

23. Okano, M. et al. Mib1 modulates dynamin 2 recruitment via Snx18 to promote Dll1 endocytosis for efficient Notch signaling. Genes Cells 21, 425-441 (2016).

24. Kawaguchi, D., Yoshimatsu, T., Hozumi, K. \& Gotoh, Y. Selection of differentiating cells by different levels of delta-like 1 among neural precursor cells in the developing mouse telencephalon. Development 135, 3849-3858 (2008).

25. Mansilla, M. C., Cybulski, L. E., Albanesi, D. \& de Mendoza, D. Control of membrane lipid fluidity by molecular thermosensors. J. Bacteriol. 186, 6681-6688 (2004).

26. Holthuis, J. C. \& Menon, A. K. Lipid landscapes and pipelines in membrane homeostasis. Nature 510, 48-57 (2014).

27. Los, D. A. \& Murata, N. Regulation of enzymatic activity and gene expression by membrane fluidity. Sci. STKE 2000, pel (2000).

28. van Meer, G., Voelker, D. R. \& Feigenson, G. W. Membrane lipids: where they are and how they behave. Nat. Rev. Mol. Cell Biol. 9, 112-124 (2008).

29. Calzada, E., Onguka, O. \& Claypool, S. M. Phosphatidylethanolamine metabolism in health and disease. Int. Rev. Cell Mol. Biol. 321, 29-88 (2016).

30. Emoto, K. et al. Redistribution of phosphatidylethanolamine at the cleavage furrow of dividing cells during cytokinesis. Proc. Natl Acad. Sci. USA 93, 12867-12872 (1996).

31. Chernomordik, L., Kozlov, M. M. \& Zimmerberg, J. Lipids in biological membrane fusion. J. Membr. Biol. 146, 1-14 (1995).

32. Emoto, K. \& Umeda, M. An essential role for a membrane lipid in cytokinesis. Regulation of contractile ring disassembly by redistribution of phosphatidylethanolamine. J. Cell Biol. 149, 1215-1224 (2000).

33. Irie, A., Yamamoto, K., Miki, Y. \& Murakami, M. Phosphatidylethanolamine dynamics are required for osteoclast fusion. Sci. Rep. 7, 46715 (2017).

34. Nomura, T., Gotoh, H. \& Ono, K. Changes in the regulation of cortical neurogenesis contribute to encephalization during amniote brain evolution. Nat. Commun. 4, 2206 (2013).

35. Yamashita, W. et al. Conserved and divergent functions of Pax6 underlie species-specific neurogenic patterns in the developing amniote brain. Development 145, dev159764 (2018).

36. Nomura, T., Yamashita, W., Gotoh, H. \& Ono, K. Species-specific mechanisms of neuron subtype specification reveal evolutionary plasticity of amniote brain development. Cell Rep. 22, 3142-3151 (2018).

37. Nomura, T., Ohtaka-Maruyama, C., Kiyonari, H., Gotoh, H. \& Ono, K. Changes in Wnt-dependent neuronal morphology underlie the anatomical diversification of neocortical homologs in amniotes. Cell Rep. 31, 107592 (2020).

38. Mizutani, K. \& Saito, T. Progenitors resume generating neurons after temporary inhibition of neurogenesis by Notch activation in the mammalian cerebral cortex. Development 132, 1295-1304 (2005)

39. Hashimoto-Torii, K. et al. Interaction between Reelin and Notch signaling regulates neuronal migration in the cerebral cortex. Neuron 60, 273-284 (2008).

40. Baek, J. H., Hatakeyama, J., Sakamoto, S., Ohtsuka, T. \& Kageyama, R. Persistent and high levels of Hes1 expression regulate boundary formation in the developing central nervous system. Development 133, 2467-2476 (2006).

41. Ishikawa, Y., Onoyama, I., Nakayama, K. I. \& Nakayama, K. Notch-dependent cell cycle arrest and apoptosis in mouse embryonic fibroblasts lacking Fbxw7. Oncogene 27, 6164-6174 (2008).

42. Weinmaster, G. \& Fischer, J. A. Notch ligand ubiquitylation: what is it good for? Dev. Cell 21, 134-144 (2011).

43. Dawaliby, R. et al. Phosphatidylethanolamine is a key regulator of membrane fluidity in eukaryotic cells. J. Biol. Chem. 291, 3658-3667 (2016).
44. Suckling, R. J. et al. Structural and functional dissection of the interplay between lipid and Notch binding by human Notch ligands. EMBO J. 36, 2204-2215 (2017)

45. Kent, C. Eukaryotic phospholipid biosynthesis. Annu. Rev. Biochem. 64, 315-343 (1995).

46. Wallis, J. G., Watts, J. L. \& Browse, J. Polyunsaturated fatty acid synthesis: what will they think of next? Trends Biochem. Sci. 27, 467 (2002).

47. Fumagalli, M. et al. Greenlandic Inuit show genetic signatures of diet and climate adaptation. Science 349, 1343-1347 (2015).

48. Montgomery, M. K., Hulbert, A. J. \& Buttemer, W. A. The long life of birds: the rat-pigeon comparison revisited. PLoS ONE 6, e24138 (2011).

49. Naudi, A. et al. Membrane lipid unsaturation as physiological adaptation to animal longevity. Front. Physiol. 4, 372 (2013).

50. Nomura, T., Hattori, M. \& Osumi, N. Reelin, radial fibers and cortical evolution: insights from comparative analysis of the mammalian and avian telencephalon. Dev. Growth Differ. 51, 287-297 (2009).

51. Martin, T. E., Auer, S. K., Bassar, R. D., Niklison, A. M. \& Lloyd, P. Geographic variation in avian incubation periods and parental influences on embryonic temperature. Evolution 61, 2558-2569 (2007).

52. Hamburger, V. \& Hamilton, H. L. A series of normal stages in the development of the chick embryo. J. Morphol. 88, 49-92 (1951).

53. Tokita, M. \& Kuratani, S. Normal embryonic stages of the chinese softshelled turtle Pelodiscus sinensis. Zool. Sci. 18, 705-715 (2001).

54. Yamashita, W., Shimizu, T. \& Nomura, T. In vitro and ex ovo culture of reptilian and avian neural progenitor cells. Methods Mol. Biol. 1650, 259-265 (2017).

55. Nomura, T. et al. The evolution of basal progenitors in the developing nonmammalian brain. Development 143, 66-74 (2016).

56. Stancik, E. K., Navarro-Quiroga, I., Sellke, R. \& Haydar, T. F. Heterogeneity in ventricular zone neural precursors contributes to neuronal fate diversity in the postnatal neocortex. J. Neurosci. 30, 7028-7036 (2010).

57. Tabata, H. \& Nakajima, K. Efficient in utero gene transfer system to the developing mouse brain using electroporation: visualization of neuronal migration in the developing cortex. Neuroscience 103, 865-872 (2001).

58. Bligh, E. G. \& Dyer, W. J. A rapid method of total lipid extraction and purification. Can. J. Biochem. Physiol. 37, 911-917 (1959).

59. Rouser, G., Siakotos, A. N. \& Fleischer, S. Quantitative analysis of phospholipids by thin-layer chromatography and phosphorus analysis of spots. Lipids 1, 85-86 (1966).

60. Matsuo, N. et al. Different mechanisms for selective transport of fatty acids using a single class of lipoprotein in Drosophila. J. Lipid Res. 60, 1199-1211 (2019).

\section{Acknowledgements}

We thank Drs. Takeshi Kawauchi and Sandra Schmid for providing the DN-dynamin 1 vector; Drs. Takeshi Kawauchi, Takuma Kumamoto, and Ikuo K. Suzuki for critically reading the manuscript; Wataru Yamashita and Kawami Misato for technical assistance; Dr. Kengo Yoshii for advice on statistical analysis. This work was supported by Japanese Grants-In-Aid for Scientific Research [KAKENHI, 16H01394 and 18H04701 (Thermal Biology), 17H03552, $21 \mathrm{H} 02591$ to T.N.] and the Uehara Memorial Foundation.

\section{Author contributions}

T.N. contributed to the experimental design, data collection, interpretation, and manuscript preparation. K.N. and M.U. contributed to lipid analyses. R.S. contributed to the data collection from cultured cells. H.G. and K.O. contributed to the experimental design, the acquisition of the reagents, and the interpretation of the data. All authors discussed the data and their interpretation and approved the final version of the manuscript.

\section{Competing interests}

The authors declare no competing interests.

\section{Additional information}

Supplementary information The online version contains supplementary material available at https://doi.org/10.1038/s41467-021-27707-5.

Correspondence and requests for materials should be addressed to Tadashi Nomura.

Peer review information Nature Communications thanks Víctor Borrell and the other, anonymous, reviewer(s) for their contribution to the peer review of this work. Peer reviewer reports are available.

Reprints and permission information is available at http://www.nature.com/reprints

Publisher's note Springer Nature remains neutral with regard to jurisdictional claims in published maps and institutional affiliations. 
(c) (i) Open Access This article is licensed under a Creative Commons Attribution 4.0 International License, which permits use, sharing, adaptation, distribution and reproduction in any medium or format, as long as you give appropriate credit to the original author(s) and the source, provide a link to the Creative Commons license, and indicate if changes were made. The images or other third party material in this article are included in the article's Creative Commons license, unless indicated otherwise in a credit line to the material. If material is not included in the article's Creative Commons license and your intended use is not permitted by statutory regulation or exceeds the permitted use, you will need to obtain permission directly from the copyright holder. To view a copy of this license, visit http://creativecommons.org/ licenses/by/4.0/.

(C) The Author(s) 2022 